\title{
Utilization of the bootstrap method for determining confidence intervals of parameters for a model of MAP1B protein transport in axons
}

\author{
I. A. Kuznetsov ${ }^{(\mathrm{a}),(\mathrm{b})}$ and A. V. Kuznetsov ${ }^{(\mathrm{c})}$
}

\begin{abstract}
This paper develops a model of axonal transport of MAP1B protein. The problem of determining parameter values for the proposed model utilizing limited available experimental data is addressed. We used a global minimum search algorithm to find parameter values that minimize the discrepancy between model predictions and published experimental results. By analyzing the best fit parameter values it was established that some processes can be dropped from the model without losing accuracy, thus a simplified version of the model was formulated. In particular, our analysis suggests that reversals in MAP1B transport are infrequent and can be neglected. The detachment of anterogradely-biased MAP1B from microtubules (MTs) and the attachment of retrogradely-biased MAP1B to MTs can also be neglected. An analytical solution for the simplified model was obtained. Confidence intervals for the determined parameters were found by bootstrapping model residuals. The resultant analysis heavily constrained the values of some parameters while showing that some could vary without significantly impacting model error. For example, our analysis suggests that, above a certain threshold value, the kinetic constant determining the rate of MAP1B transition from the retrograde pausing state to the off-track state has little impact on model error. On the contrary, the kinetic constant describing MAP1B transition from a pausing to a running state has great impact on model error.
\end{abstract}

Keywords neuron; slow axonal transport; parameter determination; resampling residuals; bootstrapping 


\section{Introduction}

One of the great challenges of mathematical modeling of biological systems is the difficulty of estimating uncertainty within such models (Kirk et al., 2015). In this paper, we suggest applying bootstrapping methodology to establish confidence intervals for estimated model parameters. We expect that the developed approach will be applicable to a wide range of biological and biophysical models.

In many models, the direct measurement of some parameters may be impossible because these parameters may describe averages of many individual processes, each involving complicated chemical and physical interactions. This necessitates fitting model predictions with experimental data. The traditional approach to model fitting is through systematic perturbation of parameters in order to match experimental data ( $\mathrm{Li}$ et al., 2014). However, it is difficult to use this approach if the model parameter space is large and can also lead to non-unique sets of model parameters.

In Kuznetsov and Kuznetsov (2016c), we determined model parameters for simulating tau protein transport in axons by finding the set of parameters that gives the best fit between model predictions and experimental measurements. Unfortunately, this methodology provides no information regarding the uncertainty of the parameters in the resultant model. Such information could be of particular importance to an experimentalist seeking to validate or apply the model. Additionally, the uncertainty can have significance for understanding the system's biological behavior, as parameters which have high certainty could correspond to physical values which require very tight control to not hamper the overall system. Similarly, parameters with large uncertainty could correspond to physical values which can vary greatly without affecting the system.

Finding model parameters that give best fit with experimental results has been previously explored; see, for example, Beck and Arnold (1977), Zadeh (2008), Zadeh and Shah (2010), Zadeh and Montas (2014), and Grytz et al. (2014). The novel feature developed in this paper was combining this method with bootstrapping in order to estimate confidence intervals of the determined parameters. We applied the developed method for estimating best fit parameters, and their confidence intervals, to a model describing MAP1B transport in an axon. We believe that the suggested approach can be generalized to many situations where model parameters (and their uncertainly) are hard to estimate directly and there are few experimental data to fit the model predictions (for example, we recently applied this method to a model of tau protein transport in Kuznetsov and Kuznetsov, 2016b). 
MAP1B is a large microtubule(MT)-associated protein with a molecular mass of more than 300 $\mathrm{kDa}$. It can also be present in soluble form. There is evidence that MAP1B is involved in MT cross-linking and stabilization, especially at the growth cone shaft. The MAP1B protein may also be involved in regulating retrograde axonal transport, specifically of mitochondria (JimenezMateos et al., 2006). There is some functional redundancy between MAP1B and tau proteins. Although MAP1B was found in Lewy bodies and it exhibits high affinity to $\alpha$-synuclein fibrils (Jensen et al., 2000), unlike tau, MAP1B is not directly involved in any specific disease (Riederer, 2007).

\section{Methods}

\subsection{Full model}

A schematic diagram showing a neuron with an axon is displayed in Fig. 1. The diagram shows that MAP1B protein can attach to MTs, detach from MTs, and participate in anterograde and retrograde transport.

Our goal is to develop a model that can be extended in the future. In our present model, we assume that MAP1B in the axon can exist in five different kinetic states, which are displayed in Fig. 2. We write the conservation of MAP1B protein in each kinetic state separately, which gives five governing equations. We used an approach similar to that which we used in developing a model for simulating a distribution of MT-associated protein tau due to its transport toward the synapse (Kuznetsov and Kuznetsov, 2015, 2016a).

In axons, the main mechanism of MAP1B traffic is slow axonal transport (Ma et al., 2000; Riederer, 2007). MAP1B is moved, anterogradely and retrogradely, due to association with molecular motors. Presumably, anterograde motion is due to association with one of the motors of the kinesin family and retrograde motion is due to association with cytoplasmic dynein. In slow axonal transport, the cargo's motion does not occur continuously; rather, short periods of motion are interrupted by long pauses. Lee and Mitchell (2015) suggested that observed pauses in cargo movement are due to motor detachment-reattachment events. Such events can be caused, for example, by the motor encountering an obstruction or reaching the end of an MT. It should be noted that the model proposed in this research simulates the behavior of the cargo (MAP1B protein), not the behavior of motors, and "pausing" in the kinetic diagram in Fig. 2 refers to the motion of cargo. 
In slow axonal transport cargo can also reverse its direction of motion, apparently due to association with different types of motors, or due to one group of motors overpowering the other groups of motors (the so-called tug-of-war model, see, for example, Mueller et al., 2008 and Hancock, 2014).

The velocity range (the particular component of slow axonal transport) at which MAP1B is transported along the axon depends on its specific isoform and also depends on the MAP1B phosphorylation state (Ma et al., 2000; Shah and Cleveland, 2002). This is because MAP1B phosphorylation determines the portion of time that a cargo complex spends moving (rather than pausing). It should also be noted that MAP1B's affinity to MTs is regulated in a complicated way, in particular, by MAP1B phosphorylation (Bonnet et al., 2001).

Some parameters required for the model can be found in the literature or estimated by a simple physical analysis. During short periods of motion MAP1B is presumably propelled by kinesin and dynein motors, whose velocities are approximately equal and can be estimated as $0.5 \mu \mathrm{m} / \mathrm{s}$ (this estimate is based on data reported in Konzack et al. (2007) for transport of tau, another MTassociated protein). In addition to the MAP1B protein associated with MTs, there is also a soluble cytoplasmic pool of MAP1B (Mansfield et al., 1992; Togel et al., 1998). MAP1B in the cytoplasmic pool must be able to diffuse (Bressloff and Newby, 2013). We performed the following estimate of diffusivity of soluble MAP1B. The mass of MAP1B is estimated to be 320$330 \mathrm{kDa}$ (Riederer, 2007) while the mass of tau protein is between $45-65 \mathrm{kDa}$, depending on the isoform (Buee et al., 2000). The diffusivity of tau protein is estimated to be $3 \mu^{2} / \mathrm{s}$ (Konzack et al., 2007). According to the Stokes-Einstein equation, diffusivity of a particle is inversely proportional to its size (Brune and Kim, 1993), so we estimated the diffusivity of soluble MAP1B protein to be approximately $0.5 \mu \mathrm{m}^{2} / \mathrm{s}$.

Also, the half-life of MAP1B is estimated to be 5.8 days $\left(5.01 \times 10^{5} \mathrm{~s}\right)$ (Riederer, 2007). Since MAP1B degradation is linked to the ubiquitin-proteasome pathway (Allen et al., 2005), we included the degradation term only in the conservation equation for free (cytoplasmic) MAP1B. This is because MAP1B must be detached from MTs in order to enter the proteolytic chamber of a proteasome.

Values of other model parameters have to be found from fitting model predictions with published experimental measurements, the most significant of which is by Black et al. (1994) who reported the distribution of MAP1B concentration along the axon. 
Summarizing, our model assumes that cargo complexes containing MAP1B can be in one of the following kinetic states: anterogradely moving, retrogradely moving, pausing during anterograde movement with kinesin motors attached (detached from an MT but maintaining close affinity to the MT), pausing during retrograde movement with dynein motors attached, or suspended freely in the cytosol in soluble form (Fig. 2). Four of our kinetic states are analogous to those proposed in the model of slow axonal transport of neurofilaments (NFs) developed in Jung and Brown (2009). (Including diffusion and protein degradation in the mathematical model of slow axonal transport was first suggested by Kuznetsov's group; see, for example, Kuznetsov et al. (2011) and Kuznetsov (2012).) As in Jung and Brown's model, we assume that MAP1B can transition from one kinetic state to another; possible transitions between kinetic states are shown by arrows in Fig. 2. Dashed lines show the transition processes that are removed from the simplified model (while they are present in the full model). Only free MAP1B can be degraded.

The equations expressing MAP1B conservation in the motor-driven states (anterograde and retrograde, respectively) are

$$
\begin{aligned}
& \frac{\partial n_{a}}{\partial t}=-v_{a} \frac{\partial n_{a}}{\partial x}-\gamma_{10} n_{a}+\gamma_{01} n_{a 0}, \\
& \frac{\partial n_{r}}{\partial t}=v_{r} \frac{\partial n_{r}}{\partial x}-\gamma_{10} n_{r}+\gamma_{01} n_{r 0},
\end{aligned}
$$

where $n_{a}=\tilde{n}_{a} / \tilde{n}_{r e f}$ and $n_{r}=\tilde{n}_{r} / \tilde{n}_{r e f}$. Dimensional MAP1B concentrations in various kinetic states (denoted by a tilde) are characterized by their linear number density, which is the number of MAP1B protein molecules residing in a particular kinetic state per unit length of the axon $(1 / \mu \mathrm{m})$. Since the governing equations and boundary conditions are linear in terms of MAP1B concentrations, the concentrations can be divided by any appropriately selected reference value ( $\left.\tilde{n}_{r e f}\right)$. This is useful because experimental data of Black et al. (1994) report fluorescence per unit volume (which is proportional to the total MAP1B concentration) rather than the absolute value of MAP1B concentration. More details about selecting a value of $\tilde{n}_{r e f}$ are provided in section

\subsection{2.}

The terms on the left-hand sides of Eqs. (1) and (2) express accumulation of anterogradely and retrogradely running $\mathrm{MAP} 1 \mathrm{~B}$, respectively; they are set to zero in the steady-state formulation. The terms involving the velocities of kinesin and dynein motors, $v_{a}$ and $v_{r}$, respectively, describe the effects of motor-driven transport. The rest of the terms are kinetic terms that describe 
the effects of MAP1B transition to/from motor-driven states (see the corresponding arrows in Fig. 2).

The equations stating MAP1B conservation in the pausing states (anterograde and retrograde, respectively) are

$\frac{\partial n_{a 0}}{\partial t}=-\left(\gamma_{01}+\gamma_{a r}+\gamma_{o f f, a}\right) n_{a 0}+\gamma_{10} n_{a}+\gamma_{r a} n_{r 0}+\gamma_{o n, a} n_{\text {free }}$,

$\frac{\partial n_{r 0}}{\partial t}=-\left(\gamma_{01}+\gamma_{r a}+\gamma_{o f f, r}\right) n_{r 0}+\gamma_{10} n_{r}+\gamma_{a r} n_{a 0}+\gamma_{o n, r} n_{f r e e}$,

where $n_{a 0}=\tilde{n}_{a 0} / \tilde{n}_{r e f}$ and $n_{r 0}=\tilde{n}_{r 0} / \tilde{n}_{r e f}$.

The terms on the left-hand sides of Eqs. (3) and (4) again express MAP1B accumulation, now in the pausing states. All terms on the right-hand sides of Eqs. (3) and (4) are kinetic terms that describe transitions to/from the pausing states (Fig. 2).

The equation expressing conservation of free MAP1B protein is

$$
\frac{\partial n_{\text {free }}}{\partial t}=D_{\text {free }} \frac{\partial^{2} n_{\text {free }}}{\partial x^{2}}+\gamma_{\text {off }, a} n_{a 0}+\gamma_{\text {off }, r} n_{r 0}-\left(\gamma_{\text {on }, a}+\gamma_{\text {on }, r}\right) n_{\text {free }}-\frac{n_{\text {free }} \ln (2)}{T_{1 / 2}},
$$

where $n_{\text {free }}=\tilde{n}_{\text {free }} / \tilde{n}_{\text {ref }}$.

The term on the left-hand side of Eq. (5) describes accumulation of soluble MAP1B protein, the term involving the diffusivity, $D_{\text {free }}$, describes effects of passive diffusion of cytosolic MAP1B, and the last term on the right-hand side describes MAP1B degradation due to MAP1B's finite half-life. The remaining terms on the right-hand side of Eq. (5) are kinetic terms that describe transitions to/from the free cytosolic state (Fig. 2).

Model variables are summarized in Table 1 and model parameters are summarized in Table 2.

Table 1. Model variables.

\begin{tabular}{|l|l|l|}
\hline Symbol & Definition & Units \\
\hline$n_{a}$ & $\begin{array}{l}\text { Dimensionless concentration of on-track } \\
\text { MAP1B moving along MTs anterogradely, } \\
\text { propelled by molecular motors, } \tilde{n}_{a} / \tilde{n}_{r e f}\end{array}$ & \\
\hline$n_{r}$ & $\begin{array}{l}\text { Dimensionless concentration of on-track } \\
\text { MAP1B moving along MTs retrogradely, }\end{array}$ & \\
\hline
\end{tabular}




\begin{tabular}{|l|l|l|}
\hline & propelled by molecular motors, $\tilde{n}_{r} / \tilde{n}_{r e f}$ & \\
\hline$n_{a 0}$ & $\begin{array}{l}\text { Dimensionless concentration of pausing on- } \\
\text { track MAP1B that is still associated with } \\
\text { molecular motors and can resume its } \\
\text { anterograde motion, } \tilde{n}_{a 0} / \tilde{n}_{r e f}\end{array}$ & $\begin{array}{l}\text { Dimensionless concentration of pausing on- } \\
\text { track MAP1B that is still associated with } \\
\text { molecular motors and can resume its retrograde } \\
\text { motion, } \tilde{n}_{r 0} / \tilde{n}_{r e f}\end{array}$ \\
\hline$n_{r 0}$ & $\begin{array}{l}\text { Dimensionless concentration of free }(\text { off-track }) \\
\text { diffusing MAP1B in the cytosol, } \tilde{n}_{f r e e} / \tilde{n}_{r e f}\end{array}$ & {$[\mathrm{~s}]$} \\
\hline$n_{\text {free }}$ & Time & {$[\mu \mathrm{m}]$} \\
\hline$t^{\text {a }}$ & Cartesian coordinate along the axon & \\
\hline$x$ &
\end{tabular}

${ }^{a}$ Note that since the equations are solved for a steady-state situation, time is not involved.

The total dimensionless concentration of MAP1B is defined as the sum of concentrations in all five kinetic states (Fig. 2):

$n_{\text {tot }}=n_{a}+n_{r}+n_{a 0}+n_{r 0}+n_{\text {free }}$.

The total flux of MAP1B has contributions from diffusion of free MAP1B as well as from motordriven transport of this protein, in anterograde and retrograde directions, respectively:

$\tilde{j}_{\text {tot }}=-D_{\text {free }} \frac{\partial \tilde{n}_{\text {free }}}{\partial x}+v_{a} \tilde{n}_{a}-v_{r} \tilde{n}_{r}$

The total dimensionless MAP1B flux is then defined as follows:

$j_{\text {tot }}=-\frac{D_{\text {free }}}{v_{a}} \frac{\partial n_{\text {free }}}{\partial x}+n_{a}-\frac{v_{r}}{v_{a}} n_{r}$

where $j_{\text {tot }}=\tilde{j}_{\text {tot }} /\left(\tilde{n}_{r e f} v_{a}\right)$.

As suggested in Kuznetsov and Kuznetsov (2015), the average MAP1B velocity (a quantity that depends on $x$ ) was then calculated as:

$v_{a v}=\frac{\tilde{j}_{t o t}}{\tilde{n}_{t o t}}=\frac{v_{a} j_{t o t}}{n_{t o t}}$ 
The percentage of MAP1B bound to MTs at a particular location in the axon is found as:

$$
\% \text { bound }=\frac{n_{a}+n_{r}+n_{a 0}+n_{r 0}}{n_{t o t}}(100 \%)
$$

\subsection{Boundary conditions}

Eqs. (1)-(5) were solved subject to the following boundary conditions:

At $x=0: \quad n_{\text {free }}=n_{\text {free }, x=0}, \quad j_{\text {tot }}=j_{\text {tot }, x=0}$.

At $x=L: \quad \frac{d n_{\text {free }}}{d x}=0, \quad j_{\text {tot }}=j_{\text {tot }, x=L}$,

where $n_{\text {free } x=0}=\tilde{n}_{\text {free }, x=0} / \tilde{n}_{r e f}, j_{t o t, x=0}=\tilde{j}_{\text {tot }, x=0} /\left(\tilde{n}_{r e f} v_{a}\right)$, and $j_{t o t, x=L}=\tilde{j}_{t o t, x=L} /\left(\tilde{n}_{r e f} v_{a}\right)$.

In Eq. (12a), we set the gradient of $n_{\text {free }}$ at $x=L$ equal to zero. There is no physical reason for $n_{\text {free }}$ to vary strongly at the end of the axon. Also, computations with other forms of the boundary condition at the axon end caused oscillatory solutions; this behavior contradicts the experimental results of Black et al. (1994).

We used the approach that Li et al. (2012) suggested to simulate NF transport in order to obtain a more detailed form of Eq. (12b). The time it takes for MAP1B protein to turn around at the terminal can be estimated as $1 / \gamma_{a r}$. Then the probability of MAP1B being degraded at the terminal is $1-\exp \left[-\log (2) /\left(\gamma_{a r} T_{1 / 2}\right)\right]$. Since the flux of MAP1B that goes into the terminal is $v_{a} \tilde{n}_{a}$, the number of MAP1B particles that are degraded in the terminal per second is

$$
\begin{aligned}
& \left(1-\exp \left[-\frac{\log (2)}{T_{1 / 2}} \frac{1}{\gamma_{a r}}\right]\right) v_{a} \tilde{n}_{a} \text {. This means that Eq. (12b) can be re-written as: } \\
& j_{t o t, x=L}=A\left(1-\exp \left[-\frac{\log (2)}{T_{1 / 2}} \frac{1}{\gamma_{a r}}\right]\right) n_{a} .
\end{aligned}
$$

Since we only estimated the rate of MAP1B degradation at the terminal, the right-hand side of Eq. (13) is multiplied by a constant $A$ whose value is determined by fitting model predictions with experimental results. Values of parameters $n_{f r e e, x=0}$ and $j_{t o t, x=0}$, which determine MAP1B concentration and flux at the hillock, respectively, are also found by determining the best fit with experimental data. 


\subsection{Numerical procedures}

\subsubsection{Solving differential equations}

Eqs. (1)-(5) were solved for an axon under steady-state or quasi-steady conditions. By quasisteady we mean the conditions when changes in the axon (for example, due to its growth) are sufficiently slow that the MAP1B concentrations effectively remain at equilibrium. For the steady-state situation Eqs. (3) and (4) become algebraic equations (AEs), while Eqs. (1), (2), and (5) become ordinary differential equations (ODEs). We eliminated $n_{a 0}(x)$ and $n_{r 0}(x)$ from Eqs. (1)-(5) by using Eqs. (3) and (4). We solved the remaining three ODEs for $n_{a}(x), n_{r}(x)$, and $n_{\text {free }}(x)$ by utilizing Matlab's BVP4C solver (Matlab R2016a, MathWorks, Natick, MA, USA). The default settings of the solver were utilized; we checked that the numerical solutions were not affected by reducing the values of error tolerance parameters, RelTol and AbsTol. After the ODEs were solved numerically, $n_{a 0}(x)$ and $n_{r 0}(x)$ were obtained from Eqs. (3) and (4).

\subsubsection{Digitizing experimental data for the MAP1B concentration}

The experimentally measured MAP1B concentration was taken from Fig. 3D of Black et al. (1994). GetData Graph Digitizer was used to scan 76 points that were in the $[0,600 \mu \mathrm{m}]$ range with respect to the distance from the neuron soma. Points corresponding to $x>600 \mu \mathrm{m}$ were not scanned since they were within the synapse. The model developed in this paper describes MAP1B transport in the axon, but does not include complicated biochemical processes which occur in the synapse.

The MAP1B concentration in Fig. 3D of Black et al. (1994) was reported in arbitrary units (they show fluorescence intensity adjusted for the volume of the axon segment where it was measured, which is proportional to the number of MAP1B molecules in a unit volume of the axon). We set the scale for the dimensionless MAP1B concentration by assigning a value of one to the dimensionless MAP1B concentration in the left most point in Fig. 3D (the point that is closest to the soma). If the total MAP1B concentration predicted by the model would exactly fit the experimental data, then the reference concentration $\tilde{n}_{r e f}$ could be interpreted as the total MAP1B concentration in the left most point in Fig. 3D of Black et al. (1994). However, as Fig. 3a shows, there is some discrepancy between the model predictions and experimental results. Therefore, the 
more accurate interpretation of $\tilde{n}_{r e f}$ would be the total MAP1B concentration in the location where $n_{t o t}$ equals to one (which, for the case displayed in Fig. 3a, is slightly to the left of the first experimental point reported by Black et al., 1994).

\subsubsection{Finding the parameter set that gives the best fit with experimental results}

The optimal values of kinetic constants and parameters characterizing fluxes of MAP1B at the boundaries were found by least square regression (LSR) (Beck and Arnold, 1977). The best fit parameters were those that minimized an objective (penalty) function, defined as the following sum of squared residuals:

$e r r=\sum_{i=1}^{N}\left(n_{t o t, i}-n_{t o t, e x p e r, i}\right)^{2}+\omega_{1} \sum_{i=1}^{N}\left(v_{a v, i}-0.0926 \frac{\mu \mathrm{m}}{\mathrm{s}}\right)^{2}+\omega_{2}\left(\% \text { bound }\left.\right|_{x=L / 2}-100\right)^{2}$

where $\omega_{1}$ and $\omega_{2}$ are the weighing factors, which were set to $10 \mathrm{~s}^{2} / \mu \mathrm{m}^{2}$ and 10 , respectively, to provide good visual agreement with experimental data points. We used $N=76$, which is the number of experimental points obtained by digitizing the data reported in Black et al. (1994). The first term on the right-hand side of Eq. (14) ensures that the distribution of the total MAP1B concentration predicted by the model is close to the one reported in Black et al. (1994). The second term constrains the MAP1B transport velocity to approximately $8 \mathrm{~mm} /$ day $(0.0926 \mu \mathrm{m} / \mathrm{s})$, which is the average of the range reported in Ma et al. (2000). The third term ensures that most of the MAP1B in the center of the axon is bound to MTs (this may not be the case close to the soma or the synapse because of reactions that MAP1B may be a part of in these locations). The value 10 for the weighting factors $\omega_{1}$ and $\omega_{2}$ was found by means of extensive numerical experimentation (data not shown). For example, if $\omega_{1}$ is set to a very large value, the predicted total MAP1B concentration strays too far from values reported in Black et al. (1994). A large value of $\omega_{1}$ would also cause overfit in terms of MAP1B velocity, because it would force the resultant MAP1B velocity to approach exactly $0.0926 \mu \mathrm{m} / \mathrm{s}$, hence ignoring the natural variance of the velocity. (The results for $\omega_{1}=1000 \mathrm{~s}^{2} / \mu \mathrm{m}^{2}$ and $\omega_{2}=10$ that display the discussed trends are shown in Figs. S1 and S2, in the Supporting Information.)

The global minimum was found by using routine MULTISTART with a local solver FMINCON, which can be found in Matlab's Optimization Toolbox. We used 5000 randomly selected starting points for the full model, and we used $10^{5}$ randomly selected starting points for the simplified 
model. Best fit values of parameters were then found by iterating from these starting points by using FMINCON. Computations were performed utilizing a Dell Precision T7810 Workstation with an Intel Xeon 2.40 GHz processor (hereafter Dell Workstation).

\subsubsection{Simplified model (for the steady-state case)}

Examination of the best fit parameter values for the full model (see column 5 in Table 2) reveals that the optimal values of the parameters $\gamma_{a r}, \gamma_{r a}, \gamma_{o n, r}$, and $\gamma_{o f f, a}$ (the corresponding rows are shaded grey in Table 2) are very close to zero. This suggests that the terms involving these four parameters can be dropped from the full model. This means that in the simplified model the kinetic processes corresponding to the arrows shown by dashed lines in Fig. 2 were removed.

For the steady-state situation the simplified model is given by the following equations. The equations for the motor-transported MAP1B (anterogradely and retrogradely, respectively) are

$$
\begin{aligned}
& 0=-v_{a} \frac{\partial n_{a}}{\partial x}-\gamma_{10} n_{a}+\gamma_{01} n_{a 0}, \\
& 0=v_{r} \frac{\partial n_{r}}{\partial x}-\gamma_{10} n_{r}+\gamma_{01} n_{r 0} .
\end{aligned}
$$

The equations for MAP1B concentrations in the pausing states (anterograde and retrograde, respectively) are

$$
\begin{aligned}
& 0=-\gamma_{01} n_{a 0}+\gamma_{10} n_{a}+\gamma_{o n, a} n_{\text {free }}, \\
& 0=-\left(\gamma_{01}+\gamma_{o f f, r}\right) n_{r 0}+\gamma_{10} n_{r} .
\end{aligned}
$$

The equation for the concentration of free MAP1B protein is

$$
0=D_{\text {free }} \frac{\partial^{2} n_{\text {free }}}{\partial x^{2}}+\gamma_{\text {off }, r} n_{r 0}-\gamma_{\text {on }, a} n_{\text {free }}-\frac{n_{\text {free }} \ln (2)}{T_{1 / 2}}
$$

The boundary conditions given by Eqs. (11a,b) and (12a,b) stand. Note that for $\gamma_{a r} \rightarrow 0$ Eq. (13) collapses to

$$
j_{t o t, x=L}=A n_{a} .
$$

Eqs. (6)-(10) stand as well. 
Equations (15)-(19) of the simplified model can be solved numerically by using the same approach that is described in section 2.3.1 for the full model. However, they also can be solved analytically; the analytical solution is given in the Appendix. The analytical solution is much (at least by two orders of magnitude) faster than the numerical solution, which was especially useful when bootstrapping, a computationally intensive statistical technique, was used to estimate confidence intervals for parameters of the simplified model (the technique is described in section 2.3.5).

A comparison between solutions of the full and simplified models is described in section 3.3; it shows that both models produce identical results.

\subsubsection{Finding confidence intervals for parameters by using resampling residuals}

To characterize the response of the model to changes in the input (experimental) data we utilized resampling residuals, a type of bootstrapping technique (Efron and Tibshirani, 1993; Davison and Hinkley, 1997). First, residuals over $N$ (in our case $N=76$ ) points were calculated as the difference between the experimentally measured values and model predictions of the total MAP1B concentration:

$\varepsilon_{i}=n_{\text {tot,exper }, i}-n_{\text {tot }, i}(i=1, \ldots, N)$.

Next, $M$ sets of new, surrogate data were generated as follows:

$n_{\text {tot }, \text { exper }, i}^{*}=n_{t o t, i}+\varepsilon_{j}$,

where an asterisk on $n$ denotes surrogate "experimental" data generated by resampling residuals. In Eq. (22), $j$ is randomly drawn (with replacement) from the list $(1, \ldots, N)$. This procedure generated $M$ (we used $M=1000$ ) new distributions of the total MAP1B concentration along the axon.

We then used the procedure described in section 2.3.3 to find the best fit parameters for the simplified model, now using surrogate data instead of real experimental data for the distribution of the MAP1B concentration along the axon. The analytical solution described in the Appendix was used when possible because it was faster than the numerical solution. However, using the analytical solution requires finding integration constants $C_{1}-C_{4}$ (see the Appendix). Sometimes Matlab's function FSOLVE, which was employed for this purpose, did not converge. In this case the code switched to the numerical solution of Eqs. (15)-(19). The described procedure, applied to 
$M$ bootstrap samples, gave $M$ sets of seven parameters for the simplified model. The obtained data were used to produce seven histograms, one for each parameter.

In order to estimate confidence intervals for the seven parameters of the simplified model, we first utilized the percentile bootstrap. $90 \%$ confidence intervals for each parameter were found by trimming 5\% from the lower and upper ends of the corresponding histogram, which was done utilizing Matlab's PRCTILE function. The major advantage of using the percentile bootstrap in our problem was the production of physically reasonable results for all seven parameters, in which the lower ends of the confidence intervals were non-negative. However, the limitation of the percentile bootstrap is the assumption that the sampling distribution is a good approximation of the unknown true population distribution.

For comparison, we also obtained estimates of parameter values and their $90 \%$ confidence intervals by the empirical bootstrap, which is used more often than the percentile bootstrap since it does not assume that the sampling distribution provides a good approximation for the population distribution. Instead, the empirical bootstrap method makes the weaker assumption that the variation of the population distribution around the mean value is well approximated by the variation of the sampling distribution around the estimate of the mean value (Hall, 1988; Singh and Xie, 2008; Chernick and LaBudde, 2011). In our implementation of the empirical bootstrap, we studied the variation of model parameters around their optimal values estimated by LSR (given in the fourth column of Table 3). In this case 5\% was trimmed from the lower and upper ends of the variance distribution.

\section{Results}

\subsection{Estimated optimal parameter values for the full model}

Column 5 in Table 2 gives parameters for the full model. The best fit values were obtained as described in section 2.3.3. We utilized 5000 randomly selected starting points, the CPU time on the Dell Workstation was 143 hours. The obtained minimum value of the objective function err, defined by Eq. (14), was 1.981998. To check independence of this result on the number of random points we compared results obtained with 2000 and 5000 random points; they produced the same minimum. 
Table 2. Model parameters and their estimation from the literature or by LSR (see footnote "b" below this table).

\begin{tabular}{|c|c|c|c|c|}
\hline Symbol & Definition & Units & $\begin{array}{l}\text { Reference } \\
\text { or } \\
\text { estimation } \\
\text { method }\end{array}$ & $\begin{array}{l}\text { Estimated } \\
\text { value }\end{array}$ \\
\hline$D_{d i f}$ & $\begin{array}{l}\text { Diffusivity of MAP1B } \\
\text { protein in the off-track state }\end{array}$ & {$\left[\mu \mathrm{m}^{2} / \mathrm{s}\right]$} & $\begin{array}{l}\text { Konzack } \\
\text { et al. } \\
(2007)^{\text {a }}\end{array}$ & 0.5 \\
\hline$L$ & Length of the axon & {$[\mu \mathrm{m}]$} & $\begin{array}{l}\text { Black et } \\
\text { al. (1994) }\end{array}$ & 600 \\
\hline$T_{1 / 2}$ & Half-life of MAP1B protein & {$[\mathrm{s}]$} & $\begin{array}{l}\text { Riederer } \\
(2007)\end{array}$ & $5.01 \times 10^{5}$ \\
\hline$v_{a}, v_{r}$ & $\begin{array}{l}\text { Velocities of kinesin and } \\
\text { dynein motors, respectively }\end{array}$ & {$[\mu \mathrm{m} / \mathrm{s}]$} & $\begin{array}{l}\text { Konzack } \\
\text { et al. } \\
(2007)\end{array}$ & $0.5,0.5$ \\
\hline$\gamma_{10}$ & $\begin{array}{l}\text { Kinetic constant describing } \\
\text { the probability of MAP1B } \\
\text { transition from the running } \\
\text { (anterograde or retrograde) to } \\
\text { the corresponding pausing } \\
\text { state }\end{array}$ & {$\left[\mathrm{s}^{-1}\right]$} & $\mathrm{LSR}^{\mathrm{b}}$ & $1.600 \times 10^{-2}$ \\
\hline$\gamma_{01}$ & $\begin{array}{l}\text { Kinetic constant describing } \\
\text { the probability of MAP1B } \\
\text { transition from the pausing } \\
\text { (anterograde or retrograde) to } \\
\text { the corresponding running } \\
\text { state }\end{array}$ & {$\left[\mathrm{s}^{-1}\right]$} & $\mathrm{LSR}^{\mathrm{b}}$ & $4.076 \times 10^{-3}$ \\
\hline$\gamma_{a r}$ & $\begin{array}{l}\text { Kinetic constant describing } \\
\text { the probability of MAP1B } \\
\text { transition from the } \\
\text { anterograde pausing to the } \\
\text { retrograde pausing state }\end{array}$ & {$\left[\mathrm{s}^{-1}\right]$} & $\mathrm{LSR}^{\mathrm{b}}$ & $6.257 \times 10^{-16 \mathrm{c}}$ \\
\hline$\gamma_{r a}$ & $\begin{array}{l}\text { Kinetic constant describing } \\
\text { the probability of MAP1B } \\
\text { transition from the retrograde } \\
\text { pausing to the anterograde } \\
\text { pausing state }\end{array}$ & {$\left[\mathrm{s}^{-1}\right]$} & $\mathrm{LSR}^{\mathrm{b}}$ & $1.453 \times 10^{-8 c}$ \\
\hline$\gamma_{o n, a}$ & $\begin{array}{l}\text { Kinetic constant describing } \\
\text { the probability of MAP1B } \\
\text { transition from the off-track } \\
\text { state to the anterograde } \\
\text { pausing state }\end{array}$ & {$\left[\mathrm{s}^{-1}\right]$} & $\mathrm{LSR}^{\mathrm{b}}$ & $6.326 \times 10^{-4}$ \\
\hline
\end{tabular}




\begin{tabular}{|l|l|l|l|l|}
\hline$\gamma_{\text {on, },}$ & $\begin{array}{l}\text { Kinetic constant describing } \\
\text { the probability of MAP1B } \\
\text { transition from the off-track } \\
\text { state to the retrograde pausing } \\
\text { state }\end{array}$ & {$\left[\mathrm{s}^{-1}\right]$} & LSR $^{\mathrm{b}}$ & $4.977 \times 10^{-10 \mathrm{c}}$ \\
\hline$\gamma_{\text {off }, a}$ & $\begin{array}{l}\text { Kinetic constant describing } \\
\text { the probability of MAP1B } \\
\text { transition from the } \\
\text { anterograde pausing state to } \\
\text { the off-track state }\end{array}$ & {$\left[\mathrm{s}^{-1}\right]$} & LSR $^{\mathrm{b}}$ & $6.257 \times 10^{-16 \mathrm{c}}$ \\
\hline$\gamma_{\text {off }, r}$ & $\begin{array}{l}\text { Kinetic constant describing } \\
\text { the probability of MAP1B } \\
\text { transition from the retrograde } \\
\text { pausing state to the off-track } \\
\text { state }\end{array}$ & {$\left[\mathrm{s}^{-1}\right]$} & LSR $^{\mathrm{b}}$ & $2.000 \times 10^{1 \mathrm{~d}}$ \\
\hline$j_{\text {tot }, x=0}$ & $\begin{array}{l}\text { Dimensionless total flux of } \\
\text { MAP1B into the axon }\end{array}$ & & LSR $^{\mathrm{b}}$ & $1.123 \times 10^{-1}$ \\
\hline$n_{\text {free, } x=0}$ & $\begin{array}{l}\text { Dimensionless concentration } \\
\text { of free (off-track) MAP1B } \\
\text { protein at the axon hillock }\end{array}$ & & LSR $^{\mathrm{b}}$ & $7.160 \times 10^{-1}$ \\
\hline$A$ & Coefficient in Eq. (13) & & LSR $^{\mathrm{b}}$ & $5.811 \times 10^{-1}$ \\
\hline
\end{tabular}

${ }^{a}$ See the analysis in section 2.1 .

${ }^{\mathrm{b}}$ Values of 11 out of 16 parameters in the full model are not readily available from the literature and cannot be easily found by simple physical analysis. Values of these 11 parameters are estimated by optimizing the fit between model predictions and published experimental results, as described in section 2.3.3.

${ }^{\mathrm{c}}$ The fact that computed best fit parameter values in the last column of the grey shaded rows are small suggests that the corresponding parameters may be dropped from the model.

${ }^{\mathrm{d}}$ The value of $20 \mathrm{~s}^{-1}$ was obtained because the search for the minimum of the objective function err (the sum of squared residuals defined by Eq. (14)) was conducted in the range $0 \leq \gamma_{\text {off }, r} \leq 20$ $\mathrm{s}^{-1}$. Further increase of $\gamma_{\text {off }, r}$ above the value of $20 \mathrm{~s}^{-1}$ (more precisely above the value of $2 \mathrm{~s}^{-1}$, as one can see in Fig. S3a) does not significantly affect parameter err. The other six parameters produce a well-defined minimum, a typical situation is shown in Fig. S3b, which displays the effect of $n_{\text {free }, x=0}$. The independence of the objective function err from further increases of $\gamma_{\text {off }, r}$ is explained as follows. The dimensionless concentration of retrograde pausing cargo, $n_{r 0}$, is 
already quite small $\left(\sim 10^{-5}\right.$, see Fig. S5b). A further increase of $\gamma_{o f f, r}$ would normally result in more transition from $n_{r 0}$ to $n_{\text {free }}$ (see the kinetic diagram in Fig. 2), but since $n_{r 0}$ is already almost zero, there is no cargo to transit, which explains why further increase of $\gamma_{\text {off }, r}$ does not affect the solution.

\subsection{Estimated optimal parameter values for the simplified model}

Values of the first 5 parameters given Table $2\left(D_{d i f}, L, T_{1 / 2}, v_{a}\right.$, and $\left.v_{r}\right)$ are the same for the full and simplified models. Best fit values of the other 7 parameters that minimize the discrepancy between model predictions and published experimental results are given in Table 3 (the procedure is described in section 2.3.3). The best fit values of the seven parameters retained in the simplified model are only slightly different from the best fit values of the same parameters in the full model. The number of random starting points was $10^{5}$, computations took 579 hours on the Dell Workstation, the obtained minimum value of the objective function err (given by Eq. (14)) was 1.982459 . This is slightly greater than the full model's err of 1.981998 , because the simplified model contains only 7 adjustable parameters while the full model contains 11 adjustable parameters. The large number of starting points was used to ensure that the global minimum is not overlooked (that the code does not end up in one of the local minima), and was possible due to availability of the analytical solution for the simplified model, which is described in the Appendix. The convergence of the solution was checked by using a smaller number $\left(10^{4}\right)$ of random starting points; the same result was obtained.

Table 3. Parameters of the simplified model found by LSR and their confidence intervals determined by the percentile and empirical bootstraps.

\begin{tabular}{|l|l|l|l|l|}
\hline Symbol & Units & $\begin{array}{l}\text { Estimated } \\
\text { value by } \\
\text { LSR }^{\mathrm{a}}\end{array}$ & $\begin{array}{l}\text { Percentile } \\
\text { bootstrap: } \\
{[90 \%} \\
\text { confidence } \\
\text { interval }]^{\mathrm{b}}\end{array}$ & $\begin{array}{l}\text { Empirical } \\
\text { bootstrap: } \\
{[90 \%} \\
\text { confidence } \\
\text { interval }]^{\mathrm{b}}\end{array}$ \\
\hline$\gamma_{10}$ & {$\left[\mathrm{~s}^{-1}\right]$} & $1.596 \times 10^{-2}$ & $\begin{array}{l}{\left[1.09 \times 10^{-2},\right.} \\
\left.2.32 \times 10^{-2}\right]\end{array}$ & $\begin{array}{l}{\left[8.74 \times 10^{-3},\right.} \\
\left.2.10 \times 10^{-2}\right]\end{array}$ \\
\hline$\gamma_{01}$ & {$\left[\mathrm{~s}^{-1}\right]$} & $4.066 \times 10^{-3}$ & $\begin{array}{l}{\left[2.74 \times 10^{-3},\right.} \\
\left.5.82 \times 10^{-3}\right]\end{array}$ & $\begin{array}{l}{\left[2.30 \times 10^{-3},\right.} \\
\left.5.39 \times 10^{-3}\right]\end{array}$ \\
\hline
\end{tabular}




\begin{tabular}{|c|c|c|c|c|}
\hline$\gamma_{o n, a}$ & {$\left[\mathrm{~s}^{-1}\right]$} & $6.290 \times 10^{-4}$ & $\begin{array}{c}6.10 \times 10^{-4}, \\
\left.1.75 \times 10^{-2}\right]\end{array}$ & $\begin{array}{l}{\left[-1.62 \times 10^{-2},\right.} \\
\left.6.48 \times 10^{-4}\right]\end{array}$ \\
\hline$\gamma_{o f f, r}$ & {$\left[\mathrm{~s}^{-1}\right]$} & $2.000 \times 10^{1}$ & $\begin{array}{l}{\left[2.15 \times 10^{0},\right.} \\
\left.1.93 \times 10^{1}\right], \\
>2.00^{\mathrm{c}}\end{array}$ & $\begin{array}{l}{\left[2.06 \times 10^{1},\right.} \\
\left.3.78 \times 10^{1}\right] \\
>2.00^{\mathrm{c}}\end{array}$ \\
\hline$j_{t o t, x=0}$ & & $1.123 \times 10^{-1}$ & $\begin{array}{c}{\left[1.08 \times 10^{-1},\right.} \\
\left.1.19 \times 10^{-1}\right]\end{array}$ & $\begin{array}{c}{\left[1.05 \times 10^{-1},\right.} \\
\left.1.16 \times 10^{-1}\right]\end{array}$ \\
\hline$n_{\text {free }, x=0}$ & & $7.169 \times 10^{-1}$ & $\begin{array}{l}{\left[3.81 \times 10^{-1},\right.} \\
\left.2.56 \times 10^{0}\right]\end{array}$ & $\begin{array}{l}{\left[-1.13 \times 10^{0},\right.} \\
\left.1.05 \times 10^{0}\right]\end{array}$ \\
\hline $\bar{A}$ & & $5.818 \times 10^{-1}$ & $\begin{array}{c}{\left[3.81 \times 10^{-1},\right.} \\
\left.6.14 \times 10^{-1}\right]\end{array}$ & $\begin{array}{c}{\left[5.50 \times 10^{-1},\right.} \\
\left.7.83 \times 10^{-1}\right]\end{array}$ \\
\hline
\end{tabular}

${ }^{a}$ The LSR procedure was similar to that utilized for the full model, the procedure is described in section 2.3.3.

${ }^{\mathrm{b}}$ Computations were performed with 1000 resamples. CPU time on the Dell Workstation was 467 hours. After histograms for the seven model parameters were computed, confidence intervals were estimated by using the percentile and empirical bootstraps, during a post-processing step.

${ }^{\mathrm{c}}$ The histogram for parameter $\gamma_{\text {off }, r}$ is not well behaved and should be interpreted with caution. The optimization procedure that produced a histogram in Fig. $7 \mathrm{~b}$ was performed assuming that $0 \leq \gamma_{o f f, r} \leq 20 \mathrm{~s}^{-1}$. The lower bound of the confidence interval that follows from this histogram is $\sim 2 \mathrm{~s}^{-1}$. The upper bound for this parameter should be set to infinity. As shown in Fig. S3a, the objective function err that characterizes the discrepancy between the model predictions and experiment becomes almost insensitive to this parameter when $\gamma_{o f f, r}$ is larger than approximately $2 \mathrm{~s}^{-1}$. The infinite upper bound for parameter $\gamma_{o f f, r}$ was confirmed by repeating the bootstrap computations assuming that $0 \leq \gamma_{\text {off }, r} \leq 10^{3} \mathrm{~s}^{-1}$ (Fig. S7) and $0 \leq \gamma_{\text {off }, r} \leq 10^{4} \mathrm{~s}^{-1}$ (data not shown).

\subsection{Comparison between solutions of the full and simplified models}

The total MAP1B concentration predicted by the model agrees very well with the experimentally measured MAP1B concentration (Fig. 3a). The model correctly captures a decrease of the MAP1B concentration in the beginning of the axon, then a region with a constant MAP1B concentration, and finally, a four-fold increase of the MAP1B concentration toward the synapse 
in the distal axon. Components of the total concentration are shown in Figs. S4-S6. For each component as well as for $n_{t o t}$ the agreement between the full and simplified models is excellent. This fact also suggests that the global minimum can be obtained using a much smaller number of starting points (rather than $10^{5}$ starting points that were used), since the minimum for the full model that depends on more parameters was obtained using only 5000 randomly selected starting points.

The percentage of MT-bound MAP1B takes its minimum value (43\%) in the beginning of the axon, then increases to almost $100 \%$, staying constant for some distance, and finally decreasing to about $50 \%$ at the synapse (Fig. 3b).

The problem is solved for steady-state. Therefore, without destruction of MAP1B the dimensionless MAP1B flux should be constant along the axon. But because we assumed that MAP1B is destroyed only in the free kinetic state (Eq. (5)), the rate of MAP1B destruction depends on the concentration of free MAP1B. Since $n_{\text {free }}$ is the largest in the distal and proximal axon (Fig. S6), this is where $j_{\text {tot }}$ decays at the fastest rate. On the other hand, in the region 150 $\mu \mathrm{m}<x<400 \mu \mathrm{m}$ the concentration of free MAP1B is almost zero (Fig. S6); hence, $j_{t o t}$ is almost independent of $x$ in this region (Fig. 4a). It should be noted that the range of the $y$-axis in Fig. 4a is very small; thus, the difference between $j_{t o t}$ predicted by the full and simplified models is also very small.

The average MAP1B velocity depends on the MAP1B flux and the total MAP1B concentration (Eq. (9)). An increase of the average velocity in the beginning of the axon (Fig. 4b) is caused by a decrease of $n_{\text {tot }}$ (Fig. 3a). At approximately $x=150 \mu \mathrm{m}$ both $j_{\text {tot }}$ and $n_{\text {tot }}$ become constant, and $v_{a v}$ becomes constant as well. A decrease of $v_{a v}$ in the distal axon starting at approximately $x=400 \mu \mathrm{m}$ (Fig. 4b) is explained by a decrease of $j_{\text {tot }}$ (Fig. 4a) and an increase of $n_{\text {tot }}$ (Fig. 3a) toward the synapse.

\subsection{Estimated confidence intervals for the simplified model}

After the simplified model was validated by comparing its results with the full model (section 3.3), we used the bootstrapping technique to estimate confidence intervals for the determined parameter values as described in section 2.3.5. The histogram of residuals, defined by Eq. (21), resembles a normal distribution, if the two outliers on the right-hand side of the histogram are neglected (Fig. 5a). (It should be noted that the bootstrapping method does not require any 
assumptions concerning the normality of the distribution.) We generated surrogate data sets as described by Eqs. (21) and (22). Five surrogate data sets (out of 1000 data sets that were used for computing the confidence intervals) are shown in Fig. 5b. Then optimal parameters were found for each of the bootstrap distributions. The distribution for parameter $j_{t o t, x=0}$ (Fig. 8a) is the closest to the normal distribution. The distributions for parameters $\gamma_{10}$ (Fig. 6a) and $\gamma_{01}$ (Fig. 6b) are asymmetric left-skewed distributions. The distributions for parameters $\gamma_{o n, a}$ (Fig. 7a) and $n_{\text {free } x=0}$ (Fig. 8b) are asymmetric right-skewed distributions. The distribution for parameter $A$ (Fig. 9) is bimodal. The distribution for parameter $\gamma_{\text {off }, r}$ (Fig. 7b) is clearly not well-behaved; the situation for this parameter requires further investigation.

We analyzed the computed histograms by using the percentile bootstrap and the empirical bootstrap. We compared confidence intervals obtained with 1000 bootstrap samples with those obtained with 500 bootstrap samples and found no significant differences. This confirmed that 1000 bootstrap samples are sufficient for estimating confidence intervals.

Column 5 in Table 3 reports 90\% confidence intervals for parameters (whose best fit values are reported in column 4) obtained by the percentile bootstrap. Note that the confidence intervals produced by this method are asymmetric with respect to the point estimate (the best fit value). Also note that the lower and upper bounds of the confidence intervals obtained by the percentile bootstrap for all parameters are positive, which is explained by the positive values for all best fit parameters for all bootstrap samples (see the histograms in Figs. 6-9). The confidence intervals (column 5) provide very valuable information. Without them the point estimates (column 4) are not meaningful, as otherwise it is unclear over what range these parameters can take their value. The last column in Table 3 reports 90\% confidence intervals computed by the empirical bootstrap. The application of the empirical bootstrap produced negative lower bounds for $\gamma_{o n, a}$ and $n_{\text {free } x=0}$, which is physically unreasonable because all model parameters must be positive constants. The mathematical reason for this result is that the histograms for these parameters are heavily skewed to the right (Figs. 7a and $8 b$ ). The widths of the confidence intervals produced by the percentile and empirical bootstraps are approximately the same. 


\section{Discussion of the results, limitations of this study and future directions}

With properly selected parameters, the developed model is capable of reproducing experimental results in terms of the total MAP1B concentration (Fig. 3a), average velocity of MAP1B transport (Fig. 4b), and the requirement that most of MAP1B must be attached to MTs (Fig. 3b).

The conclusion from Figs. 3, 4, and S4-S6 is that the simplified model is sufficient to simulate MAP1B transport in axons. This has important biological consequences. Indeed, this suggests that reversals in MAP1B transport are insignificant $\left(\gamma_{a r}\right.$ and $\gamma_{r a}$ are negligible, see the dashed lines in Fig. 2). This may indicate that complexes containing MAP1B are driven by a single molecular motor (either kinesin or dynein). Indeed, if there were opposing motors attached to the cargo, unbinding of a motor of one type would potentially lead to a reversal (Lee and Mitchell, 2015). The prediction concerning the lack of reversals in MAP1B transport should be tested in future experiments using a technique similar to that developed in Wang et al. (2000) and Wang and Brown (2001) for NFs.

The simplified model also suggests that anterogradely-biased MAP1B does not detach from MTs ( $\gamma_{\text {off }, a}$ is negligible). There is also no significant supply of new retrogradely-moving MAP1B, since there is no attachment of retrogradely-biased MAP1B to MTs ( $\gamma_{o n, r}$ is insignificant). The model predicts accumulation of MAP1B in anterogradely-biased states $\left(n_{a}\right.$, Fig. S4a and $n_{a 0}$, Fig. S5a). Biologically, this is necessary to supply the synapse with protein synthesized in the soma. The biophysical mechanism driving this process may be driven by regulation of the processivity and affinity for MTs of anterograde (kinesin) motors transporting cargo complexes containing MAP1B.

Even for the simplified model, the concentration of retrogradely running MAP1B in the region close to the axon terminal is not zero (Fig. S4b). This is because some complexes containing MAP1B reverse at the terminal by switching their motors, and return back to the axon via retrograde transport. This creates a circulation loop in the distal axon, because retrogradely-biased MAP1B transitions back to anterogradely-biased MAP1B via kinetic processes described by constants $\gamma_{o f f, r}$ and $\gamma_{o n, a}$ (Fig. 2). The concentration of free MAP1B is also not zero in the regions close to the axon hillock and terminal (Fig. S6).

Histograms of model parameters are obtained by resampling residuals. The residuals are obtained by calculating the difference between published experimental measurements and model predictions. The residuals' distribution resembles a normal distribution (Fig. 5a). Surrogate 
experimental data (bootstrap samples) are generated by randomly re-arranging residuals (Fig. 5b). A histogram showing the frequency of a given parameter is obtained by calculating best fit parameters for 1000 different surrogate "experimental" data samples. Confidence intervals have some dependence on the method of post-processing histograms of model parameters. The advantage of the percentile bootstrap was that for all parameters the lower bounds of the confidence intervals were positive. For the empirical bootstrap the lower bounds for parameters $\gamma_{o n, a}$ and $n_{\text {free }, x=0}$ were negative.

The confidence interval for parameter $\gamma_{01}$ is the narrowest, meaning that this parameter has a strong effect on model predictions. The situation is different for parameter $\gamma_{o f f, r}$. As explained in footnote "c" after Table 3, the histogram for parameter $\gamma_{\text {off }, r}$ (Fig. 7b) should be interpreted with caution. This parameter has very little effect on the discrepancy between model prediction and experimental results for $\gamma_{o f f, r}>2 \mathrm{~s}^{-1}$ (Fig. S3a). The upper bound of the histogram displayed in Fig. $7 \mathrm{~b}$ exists because we minimized the objective function err in the domain $0 \leq \gamma_{\text {off }, r} \leq 20 \mathrm{~s}^{-1}$. We tried larger domains (for example, the histogram for this parameter obtained assuming that $0 \leq \gamma_{\text {off }, r} \leq 10^{3} \mathrm{~s}^{-1}$ is shown in Fig. S7) and found that $\gamma_{\text {off }, r}$ must be larger than $2 \mathrm{~s}^{-1}$.

For parameters characterized by left-skewed distributions $\left(\gamma_{10}\right.$, Fig. 6a and $\gamma_{01}$, Fig. 6b) the mean, reported in column 4 of Table 3, is smaller than the median. Hence, over 50\% of bootstrap results are above the mean. For parameters characterized by right-skewed distributions $\left(\gamma_{o n, a}\right.$, Fig. $7 \mathrm{a}$ and $n_{\text {free }, x=0}$, Fig. $8 \mathrm{~b}$ ), the opposite is true: the mean is larger than the median. Hence, over $50 \%$ of bootstrap results are below the mean. For a parameter with a symmetric distribution ( $j_{t o t, x=0}$, Fig. 8a), values smaller or larger than the mean are equally likely. Note that the skewed nature of the distributions is accounted for via the asymmetric nature of the confidence intervals (Table 3, columns 5 and 6).

The histogram for parameter $A$ (Fig. 9) is bimodal, although the second peak (that occurs at approximately $A=0.38$ ) has a much smaller amplitude. Note that some degree of bimodality can also be found in the histograms for parameters $\gamma_{10}$ (Fig. 6a) and $\gamma_{01}$ (Fig. 6b). This has an interesting implication: a small experimental error may result in parameter shifting from the main mode to the secondary mode, which could be interpreted as a bifurcation. The bimodal shape of the histogram makes the confidence interval for $A$ wider (Table 3, column 5). More research needs to be done before the biophysical significance of the second peak can be elucidated; for 
example, one can speculate that transition from the main mode to the secondary mode may indicate transition from a normal to a pathological state.

One of fundamental difficulties of the analysis is that the variation of MAP1B velocity with the distance from the soma is not known. Incorporating this dependence into the model would lead to more accurate predictions. It should also be noted that the presented histograms do not depict interdependencies of parameters. Future research may address finding a confidence region rather than seven independent confidence intervals. The presented approach also does not allow for multiple comparisons of parameters, as would be necessary when simultaneously comparing these computed parameters to new, perhaps empirical, values.

The following questions need to be addressed in future research. How many parameters can be reliably determined by the proposed method? How sensitive is the solution to the found best fit parameter values? (This question was partly addressed in Fig. S3.) The best fit parameters are determined from the condition that they minimize the discrepancy between model predictions and experimental results. If there is a set of parameter values that gives a local minimum with a discrepancy close to the global minimum, is it possible that the biological system evolved toward the local minimum? If the number of model parameters is increased, how are the confidence intervals of parameters affected?

\section{Conclusions}

We suggested an approach that allows for the estimation of parameter values for a model that simulates transport of MAP1B protein in an axon. The approach utilizes limited experimental data available from the literature, which are not sufficient for direct determination of parameter values. Instead, we used optimization to determine the best fit parameter values for which the model gives the best agreement with experimental results. The method does not replace the determination of model parameters from direct measurements or from physically-based estimates, which are always preferred methods for finding parameter values. The novelty of the developed approach is in using the bootstrapping technique for determining confidence intervals of estimated parameter values. We applied both the percentile and empirical bootstrap to determine the confidence intervals. The disadvantage of the empirical bootstrap was the production of negative lower bounds for some parameters whose histograms were heavily skewed.

The developed method of determination of best fit parameter values should be applied with caution, especially because minimizing a discrepancy between model predictions and 
experimental results can often result in finding a local, rather than global, minimum. However, the developed method can be useful in determining whether the model is capable of matching the experimental results. A comparison between the experimental results and the best fit model prediction can also point out to what physics is missing in the model.

\section{Acknowledgment}

AVK acknowledges with gratitude the support of the National Science Foundation (award CBET1642262) and the Alexander von Humboldt Foundation through the Humboldt Research Award.

\section{Appendix}

\section{Analytical Solution for the Simplified Model}

Eqs. (17) and (18) are algebraic equations, and they can be solved for $n_{a 0}$ and $n_{r 0}$ as:

$$
\begin{aligned}
& n_{a 0}=\frac{\gamma_{10} n_{a}}{\gamma_{01}}+\frac{\gamma_{o n, a} n_{\text {free }}}{\gamma_{01}}, \\
& n_{r 0}=\frac{\gamma_{10}}{\left(\gamma_{01}+\gamma_{o f f, r}\right)} n_{r} .
\end{aligned}
$$

Eliminating $n_{a 0}$ and $n_{r 0}$ from Eqs. (16) and (19), one obtains:

$$
\begin{aligned}
& v_{r} \frac{\partial n_{r}}{\partial x}-\gamma_{10} n_{r}+\frac{\gamma_{01} \gamma_{10}}{\left(\gamma_{01}+\gamma_{o f f, r}\right)} n_{r}=0, \\
& D_{\text {free }} \frac{\partial^{2} n_{\text {free }}}{\partial x^{2}}+\frac{\gamma_{o f f, r} \gamma_{10}}{\left(\gamma_{01}+\gamma_{\text {off }, r}\right)} n_{r}-\gamma_{\text {on }, a} n_{\text {free }}-\frac{n_{\text {free }} \ln (2)}{T_{1 / 2}}=0 .
\end{aligned}
$$

Eq. (A3) can be solved for $n_{r}$. The following is obtained:

$n_{r}(x)=C_{1} \exp \left[\frac{\gamma_{10} \gamma_{o f f, r}}{v_{r}\left(\gamma_{01}+\gamma_{o f f, r}\right)} x\right]$

Using Eq. (A5) to eliminate $n_{r}$ from Eq. (A4) and solving the result for $n_{\text {free }}$, we obtained the following: 


$$
\begin{aligned}
& n_{\text {free }}(x)=\left\{\operatorname { e x p } [ - ( \frac { \gamma _ { \text { on } , a } T _ { 1 / 2 } + \operatorname { l n } ( 2 ) } { D _ { \text { free } } T _ { 1 / 2 } } ) ^ { 1 / 2 } x ] \left[2 C_{1} \gamma_{10} \gamma_{\text {off }, r} T_{1 / 2} v_{r}^{2}\left(\gamma_{01}+\gamma_{\text {off }, r}\right)\right.\right. \\
& \times \exp \left[\left(\left[\frac{\gamma_{\text {on }, a} T_{1 / 2}+\ln (2)}{D_{\text {free }} T_{1 / 2}}\right]^{1 / 2}+\frac{\gamma_{10} \gamma_{\text {off }, r}}{v_{r}\left(\gamma_{01}+\gamma_{\text {off }, r}\right)}\right) x\right]-\left(C_{2} \exp \left[2\left(\frac{\gamma_{o n, a} T_{1 / 2}+\ln (2)}{D_{\text {free }} T_{1 / 2}}\right)^{1 / 2} x\right]+C_{3}\right) \\
& \left.\left.\times\left(2 \gamma_{10}^{2} \gamma_{\text {off }, r}^{2} D_{\text {free }} T_{1 / 2}-2 v_{r}^{2}\left(\gamma_{10}+\gamma_{\text {off }, r}\right)^{2}\left(\gamma_{\text {on }, a} T_{1 / 2}+\log (2)\right)\right)\right]\right\} \\
& \times\left\{2\left[v_{r}^{2}\left(\gamma_{01}+\gamma_{\text {off }, r}\right)^{2}\left(\gamma_{o n, a} T_{1 / 2}+\ln (2)\right)-\gamma_{10}^{2} \gamma_{\text {off }, r}^{2} D_{\text {free }} T_{1 / 2}\right]\right\}^{-1} .
\end{aligned}
$$

Eliminating $n_{a 0}$ from Eqs. (15), one obtains:

$$
-v_{a} \frac{\partial n_{a}}{\partial x}+\gamma_{o n, a} n_{\text {free }}=0
$$

Eq. (A6) is then used to eliminate $n_{\text {free }}$ from Eq. (A7), and the result is solved for $n_{a}$. The following is obtained:

$$
\begin{aligned}
& n_{a}(x)=\left\{\gamma _ { o n , a } \operatorname { e x p } [ - ( \frac { \gamma _ { \text { on } , a } T _ { 1 / 2 } + \operatorname { l n } ( 2 ) } { D _ { \text { free } } T _ { 1 / 2 } } ) ^ { 1 / 2 } x ] \left[\left(\frac{D_{\text {free }} T_{1 / 2}}{\gamma_{\text {on }, a} T_{1 / 2}+\ln (2)}\right)^{1 / 2}\left(C_{3}-C_{2} \exp \left[2\left(\frac{\gamma_{\text {on }, a} T_{1 / 2}+\ln (2)}{D_{\text {free }} T_{1 / 2}}\right)^{1 / 2} x\right]\right)\right.\right. \\
& \times\left[v_{r}^{2}\left(\gamma_{01}+\gamma_{\text {off }, r}\right)^{2}\left(\gamma_{\text {on }, a} T_{1 / 2}+\ln (2)\right)-\gamma_{10}^{2} \gamma_{\text {off }, r}^{2} D_{\text {free }} T_{1 / 2}\right]-C_{1} T_{1 / 2} v_{r}^{3}\left(\gamma_{01}+\gamma_{\text {off }, r}\right)^{2} \\
& \left.\left.\times \exp \left\{\left[\left(\frac{\gamma_{\text {on }, a} T_{1 / 2}+\ln (2)}{D_{\text {free }} T_{1 / 2}}\right)^{1 / 2}+\frac{\gamma_{10} \gamma_{\text {off }, r}}{v_{r}\left(\gamma_{01}+\gamma_{\text {off }, r}\right)}\right] x\right\}\right]\right\} \\
& \times\left\{v_{a}\left(\gamma_{10}^{2} \gamma_{\text {off }, r}^{2} D_{\text {free }} T_{1 / 2}-v_{r}^{2}\left(\gamma_{01}+\gamma_{o f f, r}\right)^{2}\left(\gamma_{o n, a} T_{1 / 2}+\ln (2)\right)\right)\right\}^{-1}+C_{4} .
\end{aligned}
$$

Integration constants $C_{1}-C_{4}$ are found by substituting Eqs. (A5), (A6), and (A8) into boundary conditions (11a,b) and (12a,b). Note that the full form of Eq. (12b) is now given by Eq. (20). The resulting algebraic equations are solved numerically by using Matlab's function FSOLVE. Although the last step of this procedure involves numerical solution, it is still much faster than direct numerical solution of Eqs. (15)-(19). 


\section{References}

Allen, E., Ding, J.Q., Wang, W., Pramanik, S., Chou, J., Yau, V., Yang, Y.M., 2005. Gigaxonincontrolled degradation of MAP1B light chain is critical to neuronal survival. Nature 438, 224-228.

Beck, J.V. and Arnold, K.J., 1977. Parameter Estimation in Science and Engineering, Wiley, New York.

Black, M.M., Slaughter, T., Fischer, I., 1994. Microtubule-associated protein-1b (MAP1b) is concentrated in the distal region of growing axons. Journal of Neuroscience 14, 857-870.

Bonnet, C., Boucher, D., Lazereg, S., Pedrotti, B., Islam, K., Denoulet, P., Larcher, J.C., 2001. Differential binding regulation of microtubule-associated proteins MAP1A, MAP1B, and MAP2 by tubulin polyglutamylation. Journal of Biological Chemistry 276, 12839-12848.

Bressloff, P.C., Newby, J.M., 2013. Stochastic models of intracellular transport. Reviews of Modern Physics 85, 135-196.

Brune, D., Kim, S., 1993. Predicting protein diffusion-coefficients. Proceedings of the National Academy of Sciences of the United States of America 90, 3835-3839.

Buee, L., Bussiere, T., Buee-Scherrer, V., Delacourte, A., Hof, P.R., 2000. Tau protein isoforms, phosphorylation and role in neurodegenerative disorders. Brain Research Reviews 33, 95130.

Chernick, M.R. and LaBudde, R.A., 2011. An Introduction to Bootstrap Methods with Applications to R, Wiley, Hoboken, NJ.

Davison, A.C. and Hinkley, D.V., 1997. Bootstrap Methods and their Application, Cambridge University Press, Cambridge.

Efron, B. and Tibshirani, R., 1993. An Introduction to the Bootstrap, Chapman \& Hall/CRC, Boca Raton, FL.

Grytz, R., Fazio, M.A., Girard, M.J.A., Libertiaux, V., Bruno, L., Gardiner, S., Girkin, C.A., Downs, J.C., 2014. Material properties of the posterior human sclera. Journal of the Mechanical Behavior of Biomedical Materials 29, 602-617.

Hall, P., 1988. Theoretical comparison of bootstrap confidence intervals. Annals of Statistics 16, 927-953.

Hancock, W.O., 2014. Bidirectional cargo transport: Moving beyond tug of war. Nature Reviews Molecular Cell Biology 15, 615-628.

Jensen, P.H., Islam, K., Kenney, J., Nielsen, M.S., Power, J., Gai, W.P., 2000. Microtubuleassociated protein 1B is a component of cortical lewy bodies and binds alpha-synuclein filaments. Journal of Biological Chemistry 275, 21500-21507. 
Jimenez-Mateos, E.M., Gonzalez-Billault, C., Dawson, H.N., Vitek, M.P., Avila, J., 2006. Role of MAP1B in axonal retrograde transport of mitochondria. Biochemical Journal 397, 53-59.

Jung, P., Brown, A., 2009. Modeling the slowing of neurofilament transport along the mouse sciatic nerve. Physical Biology 6, 046002.

Kirk, P.D.W., Babtie, A.C., Stumpf, M.P.H., 2015. Systems biology (un)certainties. Science 350, 386-388.

Konzack, S., Thies, E., Marx, A., Mandelkow, E.M., Mandelkow, E., 2007. Swimming against the tide: Mobility of the microtubule-associated protein tau in neurons. Journal of Neuroscience 27, 9916-9927.

Kuznetsov, A.V., 2012. An exact solution describing slow axonal transport of cytoskeletal elements: Effect of a finite half-life. Proceedings of the Royal Society A-Mathematical Physical and Engineering Sciences 468, 3384-3397.

Kuznetsov, A.V., Avramenko, A.A., Blinov, D.G., 2011. Investigation of the role of diffusivity on spreading, rate, and merging of the bell-shaped waves in slow axonal transport. International Journal for Numerical Methods in Biomedical Engineering 27, 1040-1053.

Kuznetsov, I.A., Kuznetsov, A.V., 2015. A comparison between the diffusion-reaction and slow axonal transport models for predicting tau distribution along an axon. Mathematical Medicine and Biology 32, 263-283.

Kuznetsov, I.A., Kuznetsov, A.V., 2016a. Can numerical modeling help understand the fate of tau protein in the axon terminal? Computer Methods in Biomechanics and Biomedical Engineering 19, 115-125.

Kuznetsov, I.A., Kuznetsov, A.V., 2016b. Simulating tau transport in an axon: Using bootstrapping for estimating confidence intervals of best fit parameter values obtained from indirect experimental data. Proceedings of the Royal Society B, submitted.

Kuznetsov, I.A., Kuznetsov, A.V., 2016c. What mechanisms of tau protein transport could be responsible for the inverted tau concentration gradient in degenerating axons? Mathematical Medicine and Biology, online first, doi: 10.1093/imammb/dqv041.

Lee, R.H., Mitchell, C.S., 2015. Axonal transport cargo motor count versus average transport velocity: Is fast versus slow transport really single versus multiple motor transport? Journal of Theoretical Biology 370, 39-44.

Li, Y., Brown, A., Jung, P., 2014. Deciphering the axonal transport kinetics of neurofilaments using the fluorescence photoactivation pulse-escape method. Physical Biology 11, 026001.

Li, Y., Jung, P., Brown, A., 2012. Axonal transport of neurofilaments: A single population of intermittently moving polymers. Journal of Neuroscience 32, 746-758. 
Ma, D., Himes, B., Shea, T., Fischer, I., 2000. Axonal transport of microtubule-associated protein 1B (MAP1B) in the sciatic nerve of adult rat: Distinct transport rates of different isoforms. Journal of Neuroscience 20, 2112-2120.

Maday, S., Twelvetrees, A.E., Moughamian, A.J., Holzbaur, E.L.F., 2014. Axonal transport: Cargo-specific mechanisms of motility and regulation. Neuron 84, 292-309.

Mansfield, S.G., Diaz-Nido, J., Gordon-Weeks, P.R., Avila, J., 1992. The distribution and phosphorylation of the microtubule-associated protein MAP 1B in growth cones. Journal of Neurocytology 21, 1007-1022.

Mueller, M.J.I., Klumpp, S., Lipowsky, R., 2008. Tug-of-war as a cooperative mechanism for bidirectional cargo transport by molecular motors. Proceedings of the National Academy of Sciences of the United States of America 105, 4609-4614.

Riederer, B.M., 2007. Microtubule-associated protein 1B, a growth-associated and phosphorylated scaffold protein. Brain Research Bulletin 71, 541-558.

Shah, J.V., Cleveland, D.W., 2002. Slow axonal transport: Fast motors in the slow lane. Current Opinion in Cell Biology 14, 58-62.

Singh, K., Xie, M., 2008. Bootstrap: A statistical method. Unpublished Manuscript, Rutgers University, USA, retrieved from 〈http://www.stat.rutgers.edu/home/ mxie/RCPapers/bootstrap.pdf).

Togel, M., Wiche, G., Propst, F., 1998. Novel features of the light chain of microtubuleassociated protein MAP1B: Microtubule stabilization, self interaction, actin filament binding, and regulation by the heavy chain. Journal of Cell Biology 143, 695-707.

Wang, L., Brown, A., 2001. Rapid intermittent movement of axonal neurofilaments observed by fluorescence photobleaching. Molecular Biology of the Cell 12, 3257-3267.

Wang, L., Ho, C.L., Sun, D.M., Liem, R.K.H., Brown, A., 2000. Rapid movement of axonal neurofilaments interrupted by prolonged pauses. Nature Cell Biology 2, 137-141.

Zadeh, K.S., 2008. Parameter estimation in flow through partially saturated porous materials. Journal of Computational Physics 227, 10243-10262.

Zadeh, K.S., Montas, H.J., 2014. Parametrization of flow processes in porous media by multiobjective inverse modeling. Journal of Computational Physics 259, 390-401.

Zadeh, K.S., Shah, S.B., 2010. Mathematical modeling and parameter estimation of axonal cargo transport. Journal of Computational Neuroscience 28, 495-507. 


\section{Figure captions}

Fig. 1. A schematic diagram showing a neuron with an axon, and a coordinate system in the axon. MAP1B protein molecules bound to MTs and suspended in the cytosol are also shown.

Fig. 2. A kinetic diagram showing five possible kinetic states for MAP1B protein and kinetic processes between these states. Four of these kinetic states are identical to the kinetic states in a slow axonal transport model for NFs (see Fig. 4 of Jung and Brown, 2009 and Fig. 1 of Li et al., 2012). Dashed lines indicate the kinetic processes that are neglected in the simplified model.

Fig. 3. (a) The dimensionless total concentration of MAP1B vs position in the axon. Open circles show experimental data from Fig. 3D of Black et al. (1994). Since Fig. 3D of Black et al. (1994) shows MAP1B concentrations in arbitrary units, we rescaled data from this figure such that the experimentally measured MAP1B concentration in the most leftward point (the closest to the soma) was equal to one. (b) Percentage of MT-bound MAP1B vs position in the axon. $\left(\omega_{1}=10\right.$ $\left.\mathrm{s}^{2} / \mu \mathrm{m}^{2}, \omega_{2}=10\right)$.

Fig. 4. (a) Total dimensionless MAP1B flux vs position in the axon. (b) MAP1B average velocity vs position in the axon. The grey shaded band indicated the range of MAP1B velocity reported in Ma et al. (2000). $\left(\omega_{1}=10 \mathrm{~s}^{2} / \mu \mathrm{m}^{2}, \omega_{2}=10\right)$.

Fig. 5. (a) Histogram showing frequency of 76 residuals which are defined by Eq. (21); the residuals show the difference between the true experimental data reported in Fig. 3D of Black et al. (1994) and model predictions for the total MAP1B concentration, computed for the best-fit parameter set. (b) $n_{t o t}$ vs $x$. Open circles show the original experimental data used to obtain the optimal set of best fit parameters given in Table 3 while crosses show $n_{\text {tot }}$ for five different bootstrap realizations. In computing the confidence intervals we used 1000 different bootstrap realizations, but showing all them would make the figure too busy.

Fig. 6. Histogram showing frequency of parameters (a) $\gamma_{10}$ and (b) $\gamma_{01}$ for different bootstrap realizations in each interval denoted with a bar. $\left(\omega_{1}=10 \mathrm{~s}^{2} / \mu \mathrm{m}^{2}, \omega_{2}=10\right)$.

Fig. 7. Histogram showing frequency of parameters (a) $\gamma_{o n, a}$ and (b) $\gamma_{o f f, r}$ for different bootstrap realizations in each interval denoted with a bar. $\left(\omega_{1}=10 \mathrm{~s}^{2} / \mu \mathrm{m}^{2}, \omega_{2}=10\right)$.

Fig. 8. Histogram showing frequency of parameters (a) $j_{t o t, x=0}$ and (b) $n_{\text {free } x=0}$ for different bootstrap realizations in each interval denoted with a bar. $\left(\omega_{1}=10 \mathrm{~s}^{2} / \mu \mathrm{m}^{2}, \omega_{2}=10\right)$. 
Fig. 9. Histogram showing frequency of parameter $A$ for different bootstrap realizations in each interval denoted with a bar. $\left(\omega_{1}=10 \mathrm{~s}^{2} / \mu \mathrm{m}^{2}, \omega_{2}=10\right)$. 


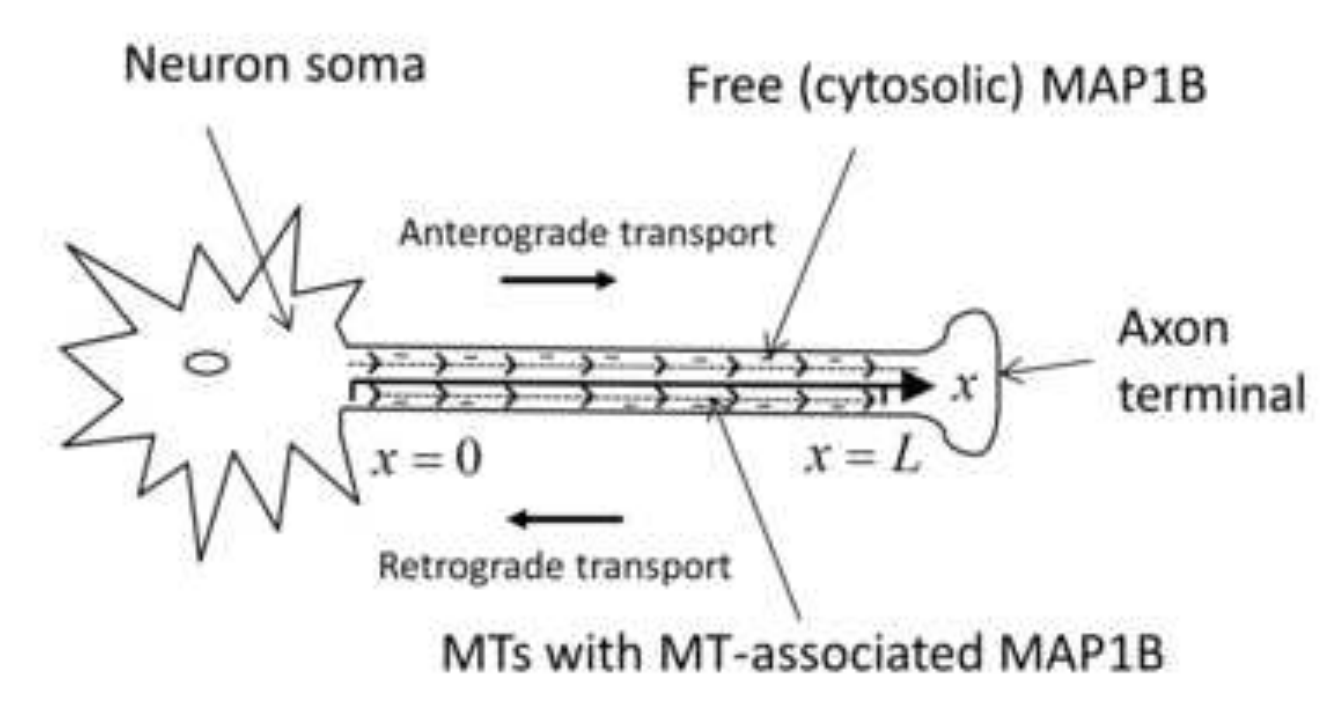

MTs with MT-associated MAP1B

Free (cytosolic) MAP1B terminal

Neuron soma

Retrograde transport

.

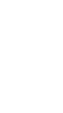

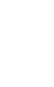


4. Figure 1

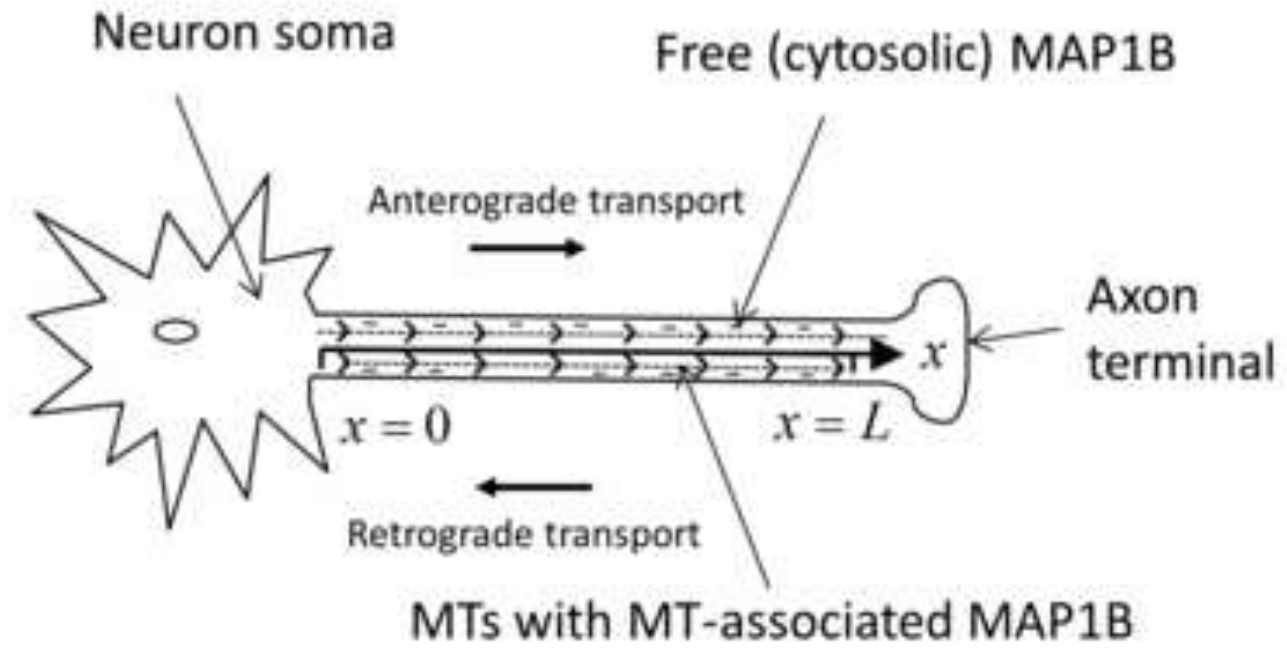




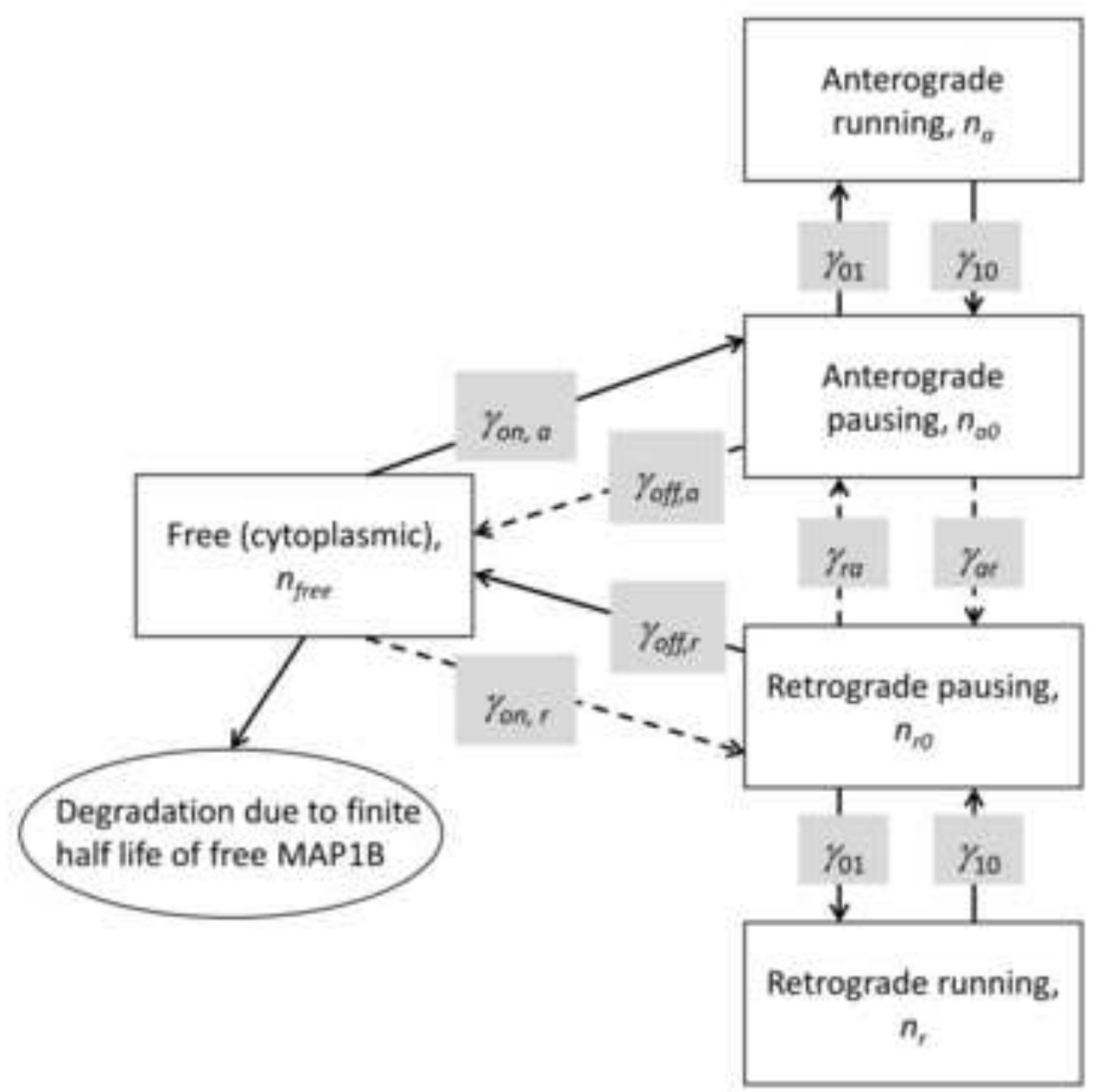




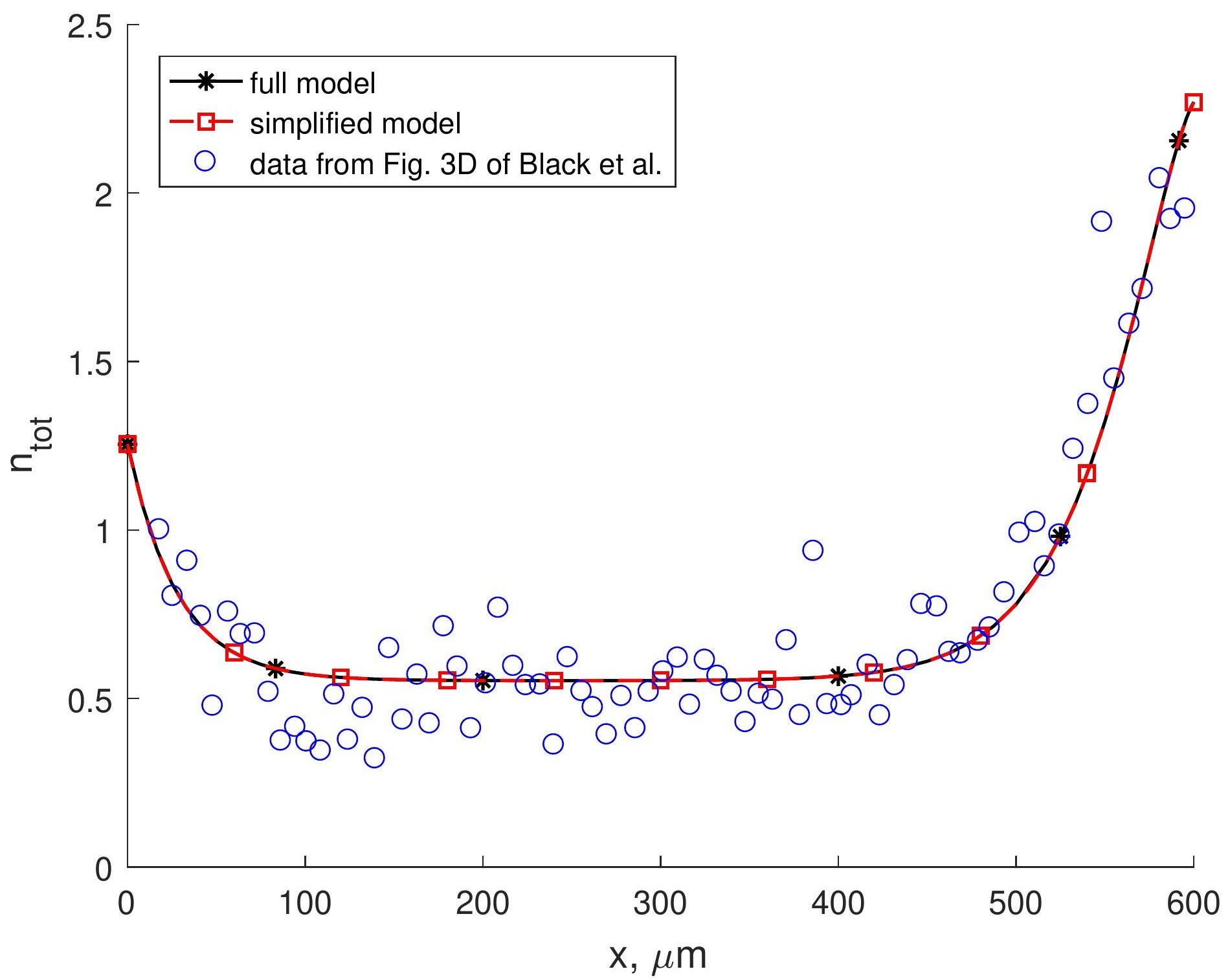




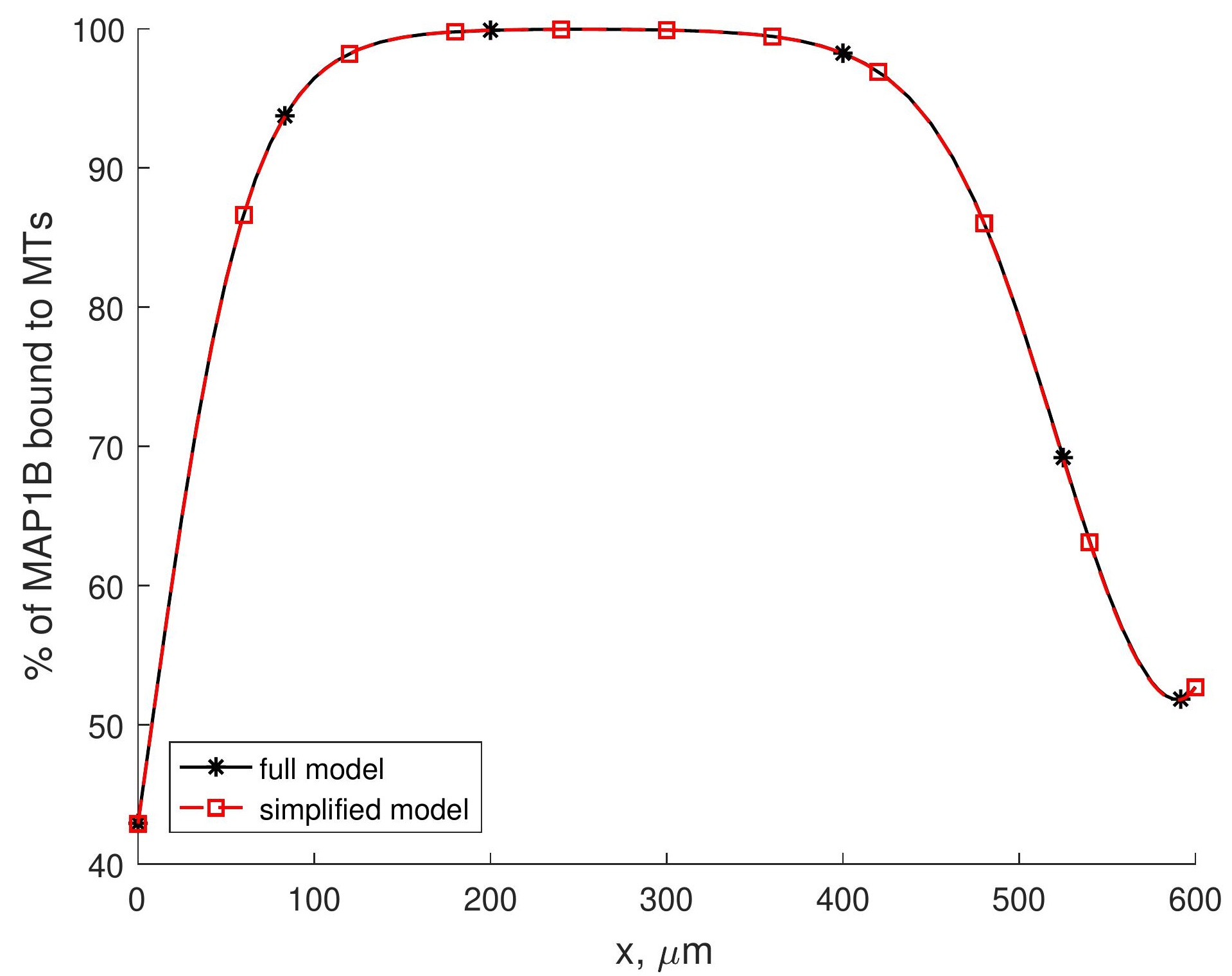




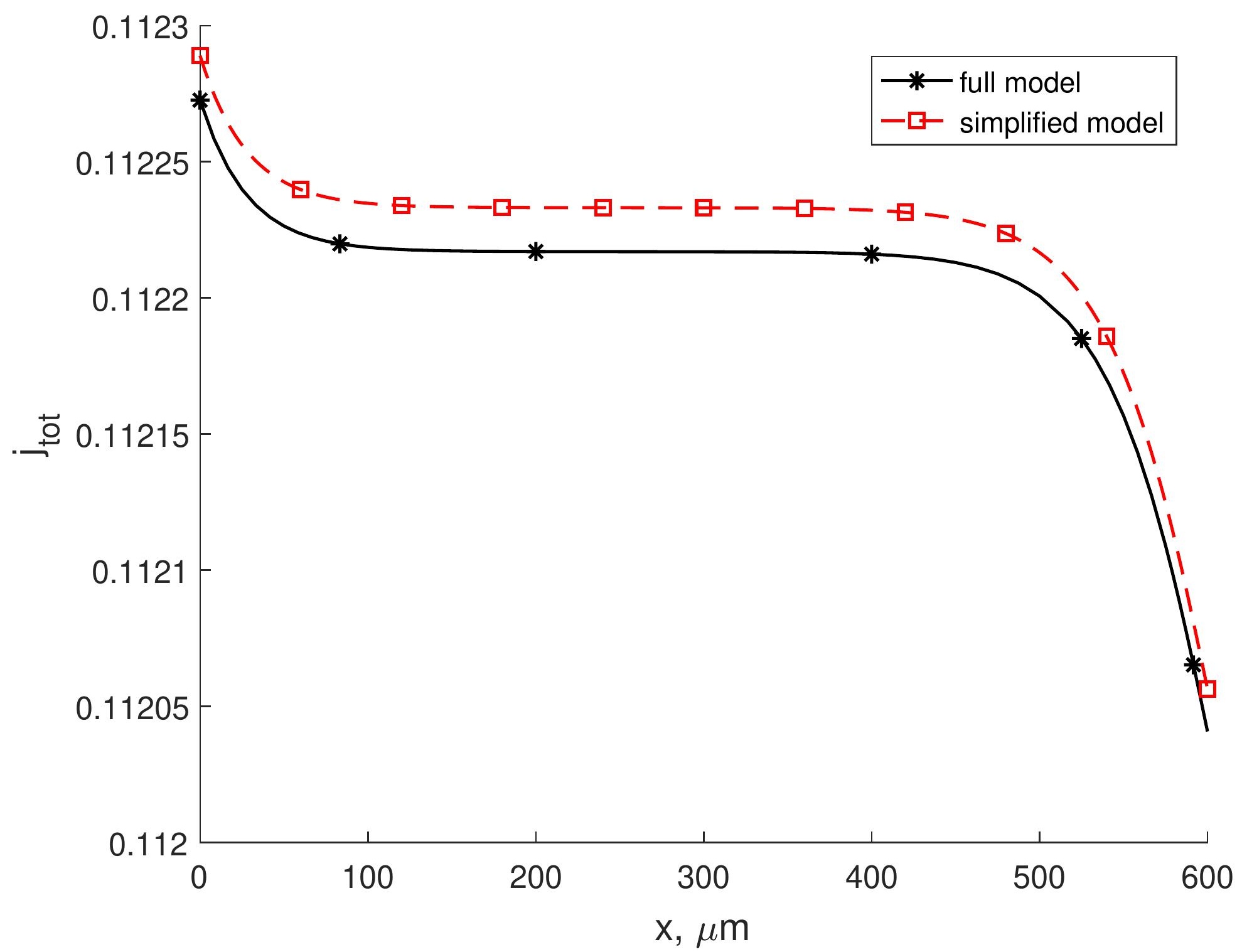




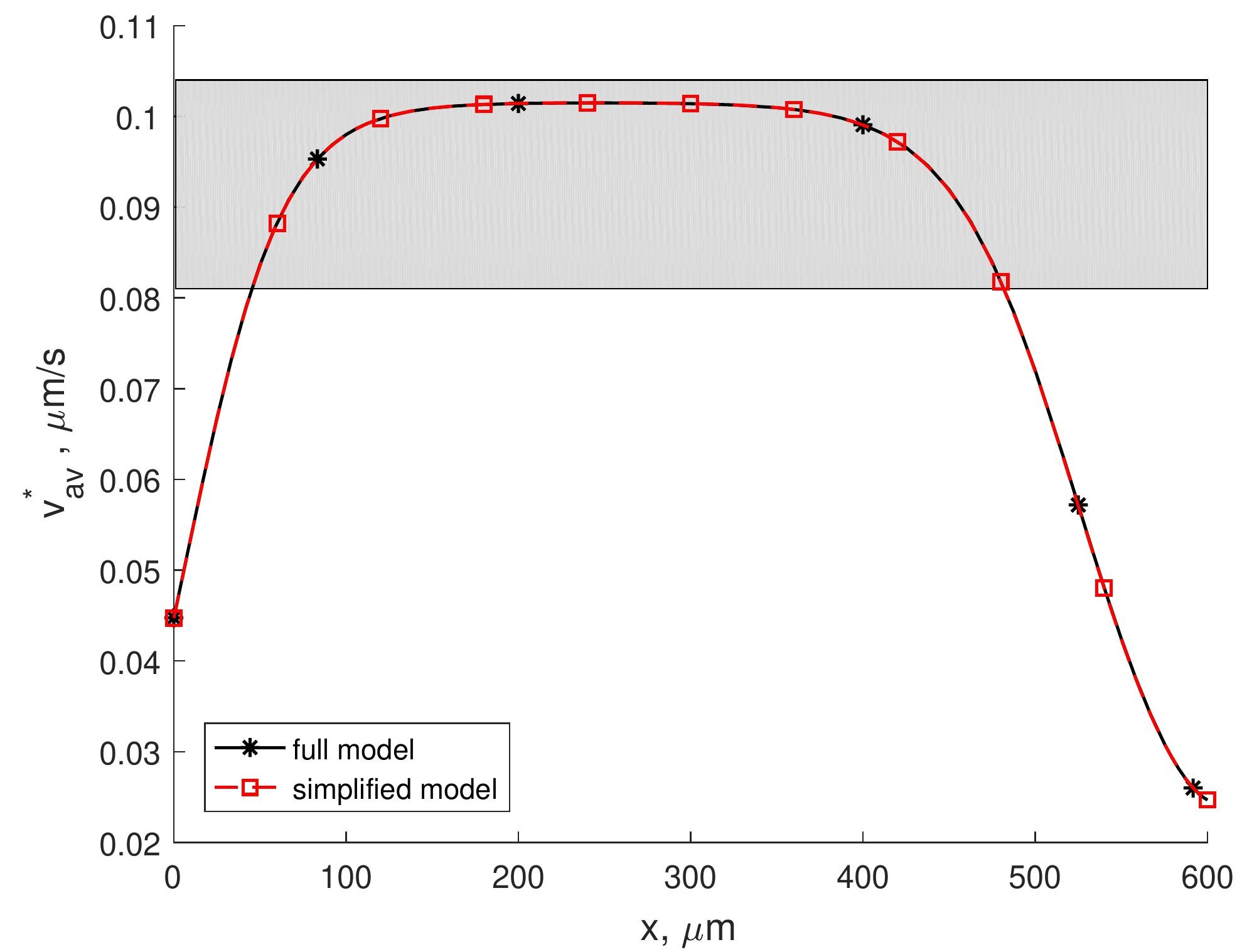




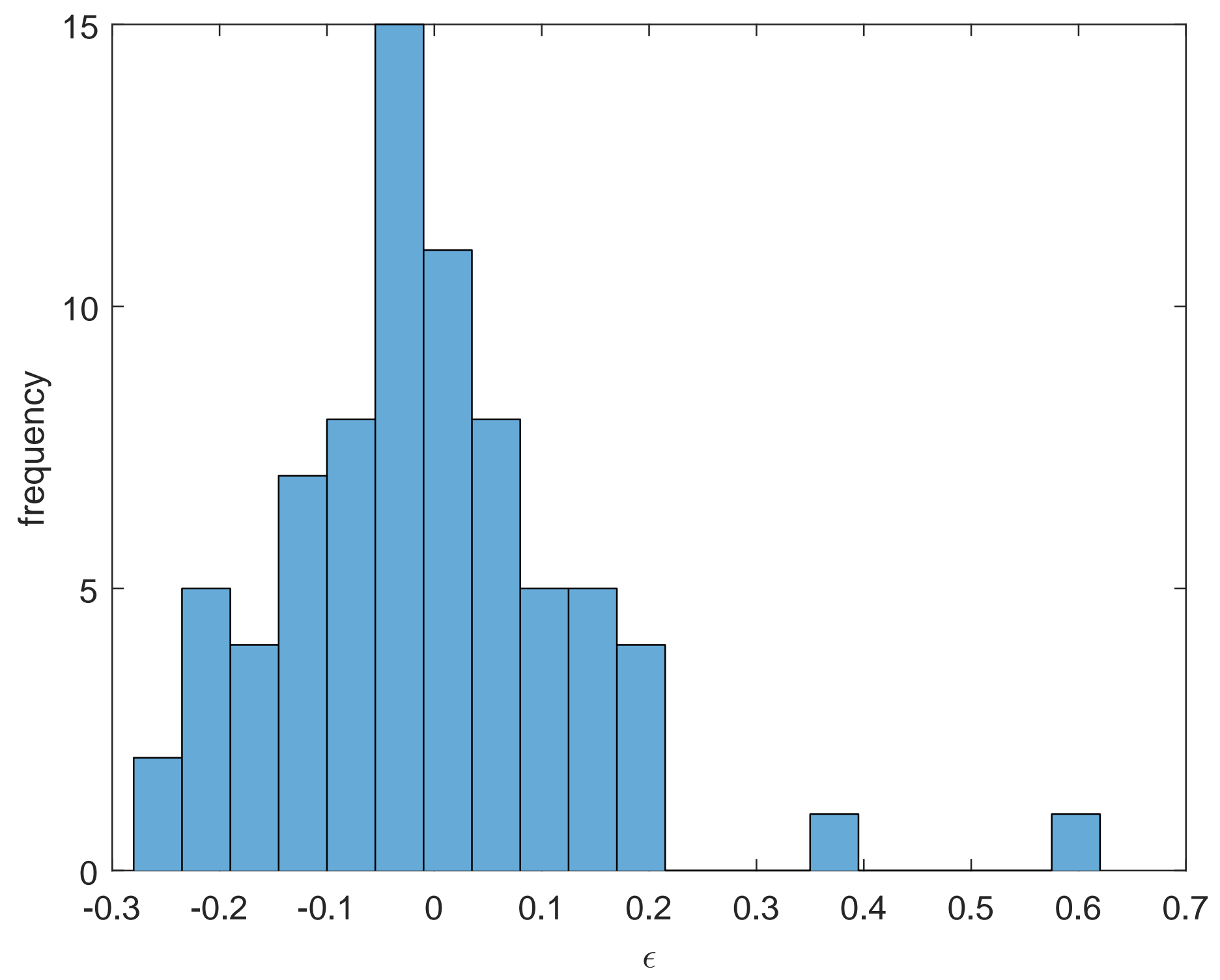




\section{Figure 5b}

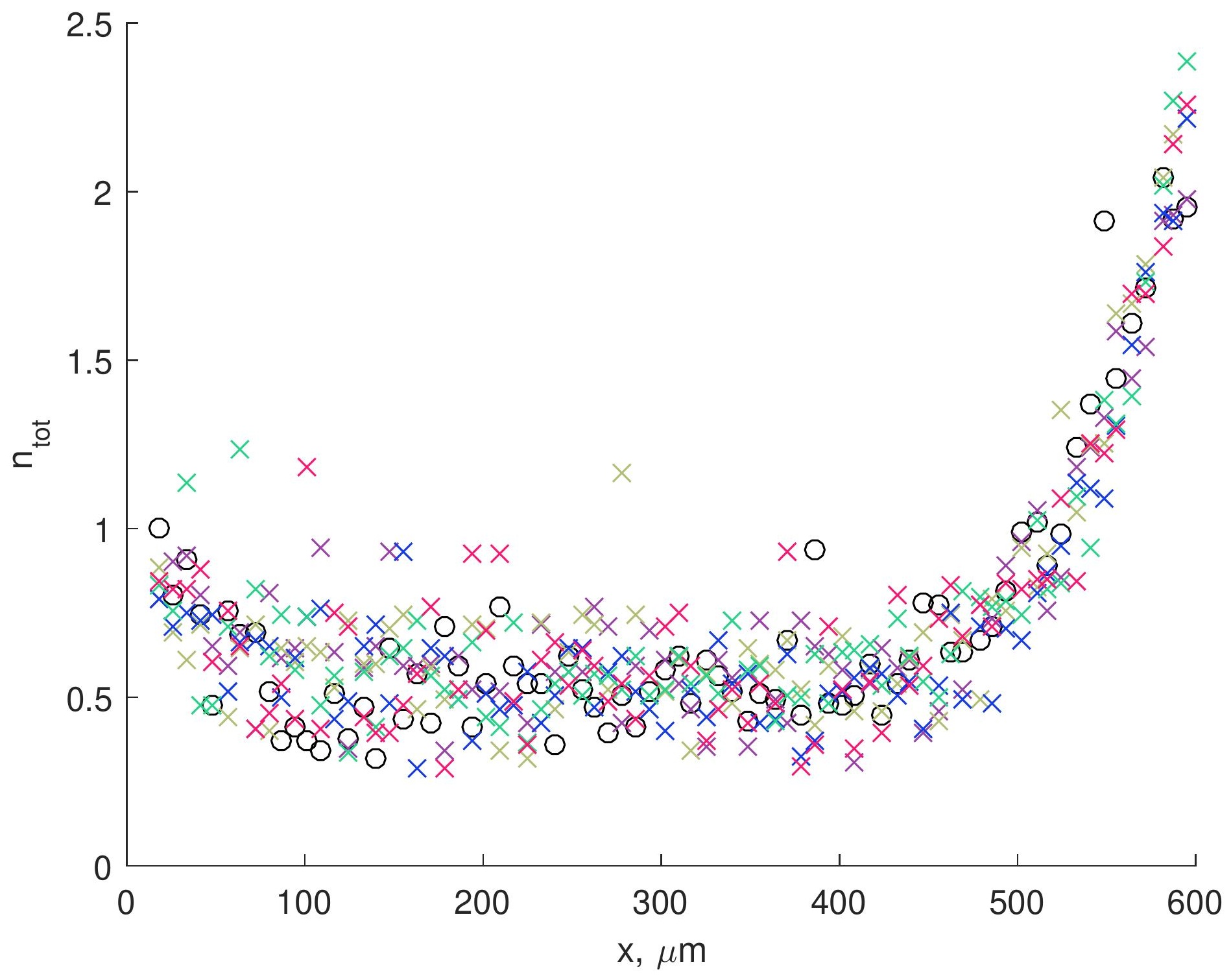




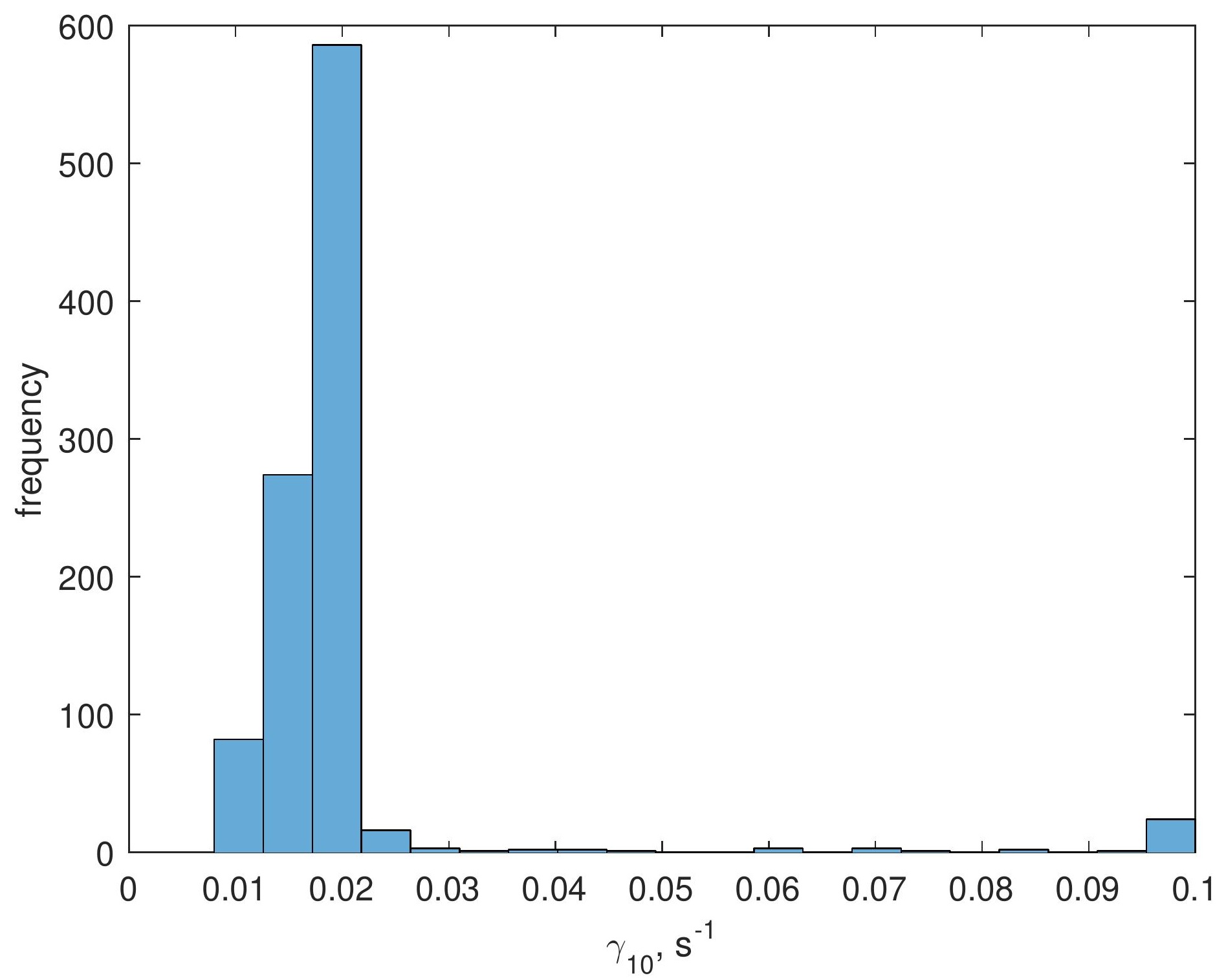




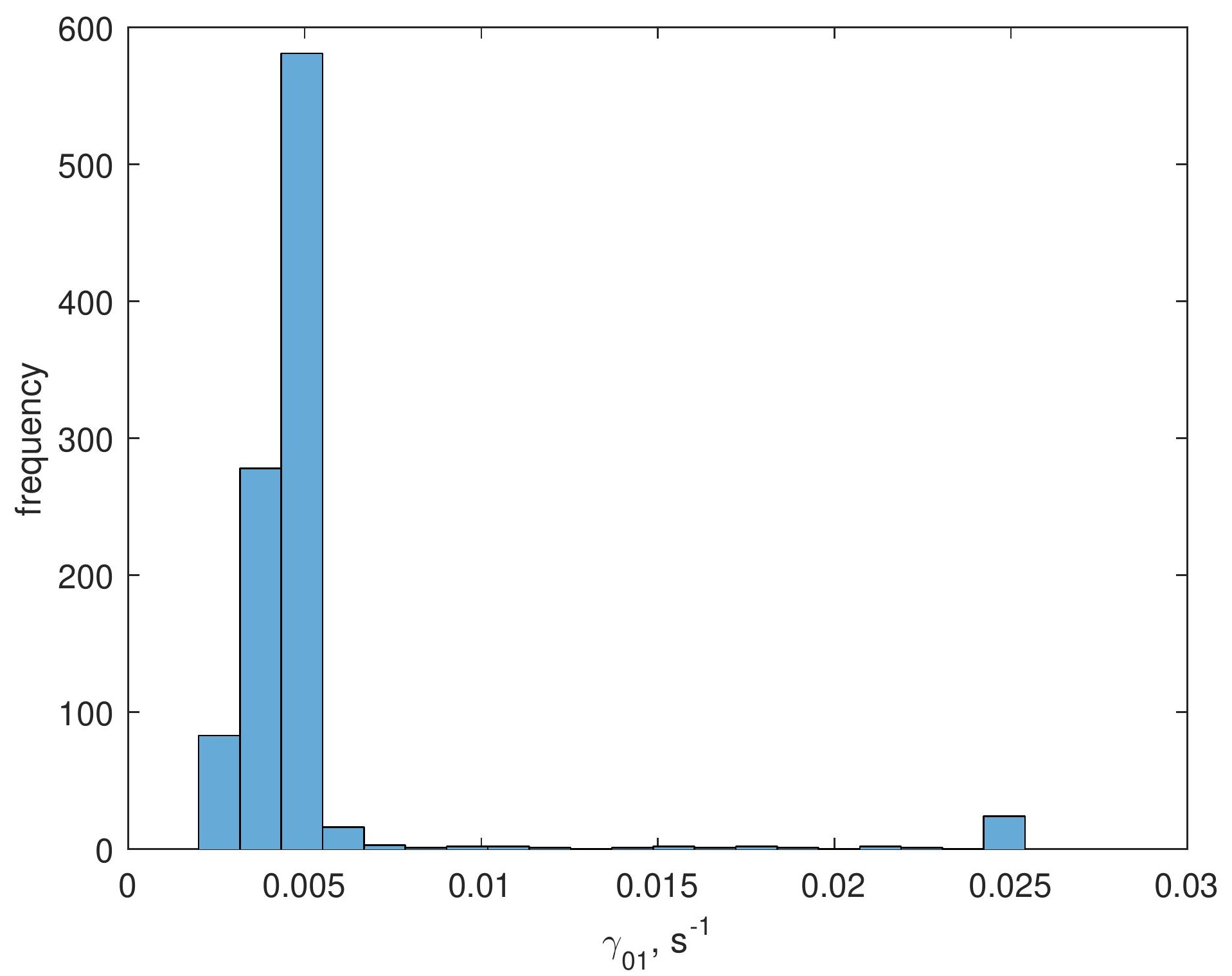




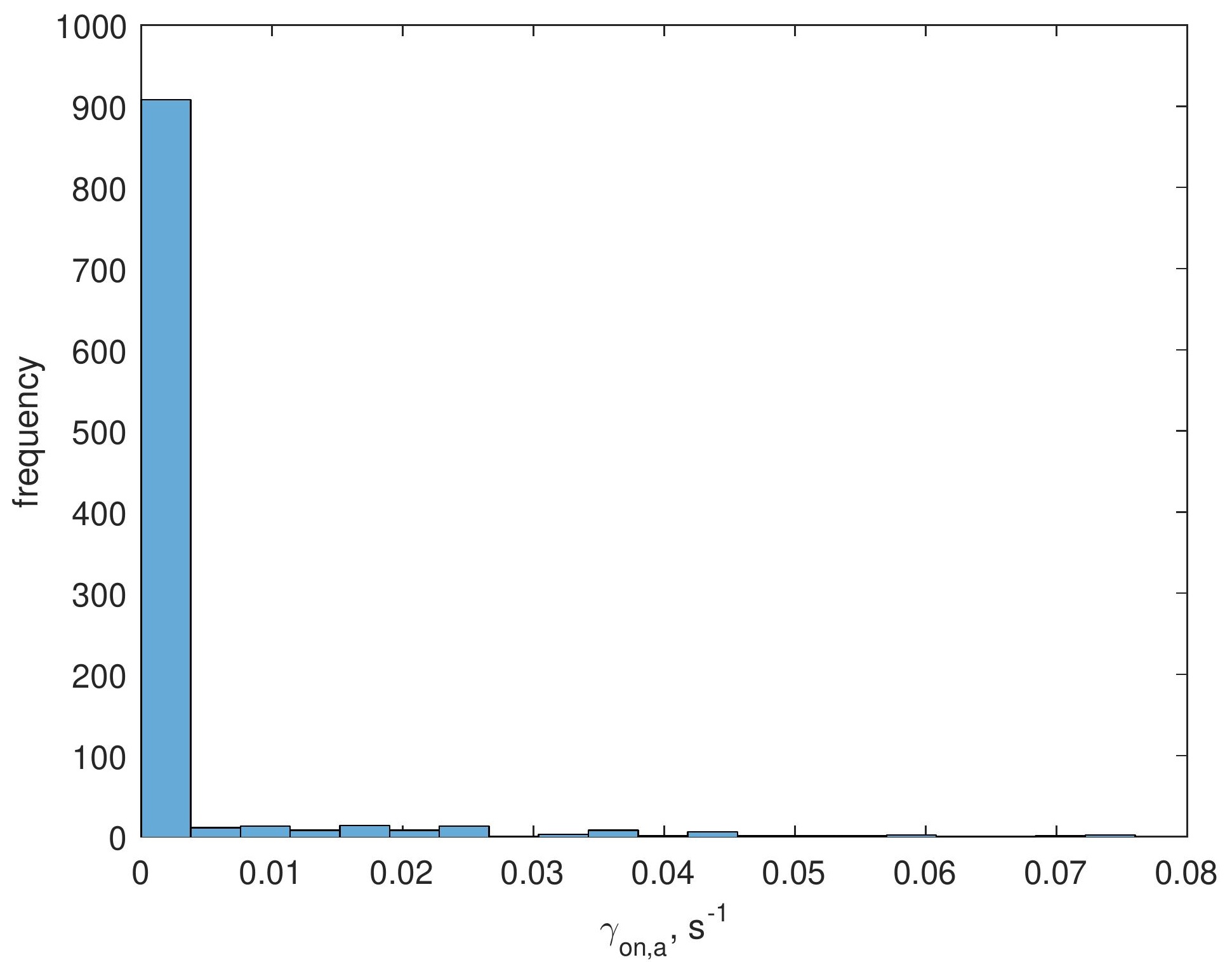




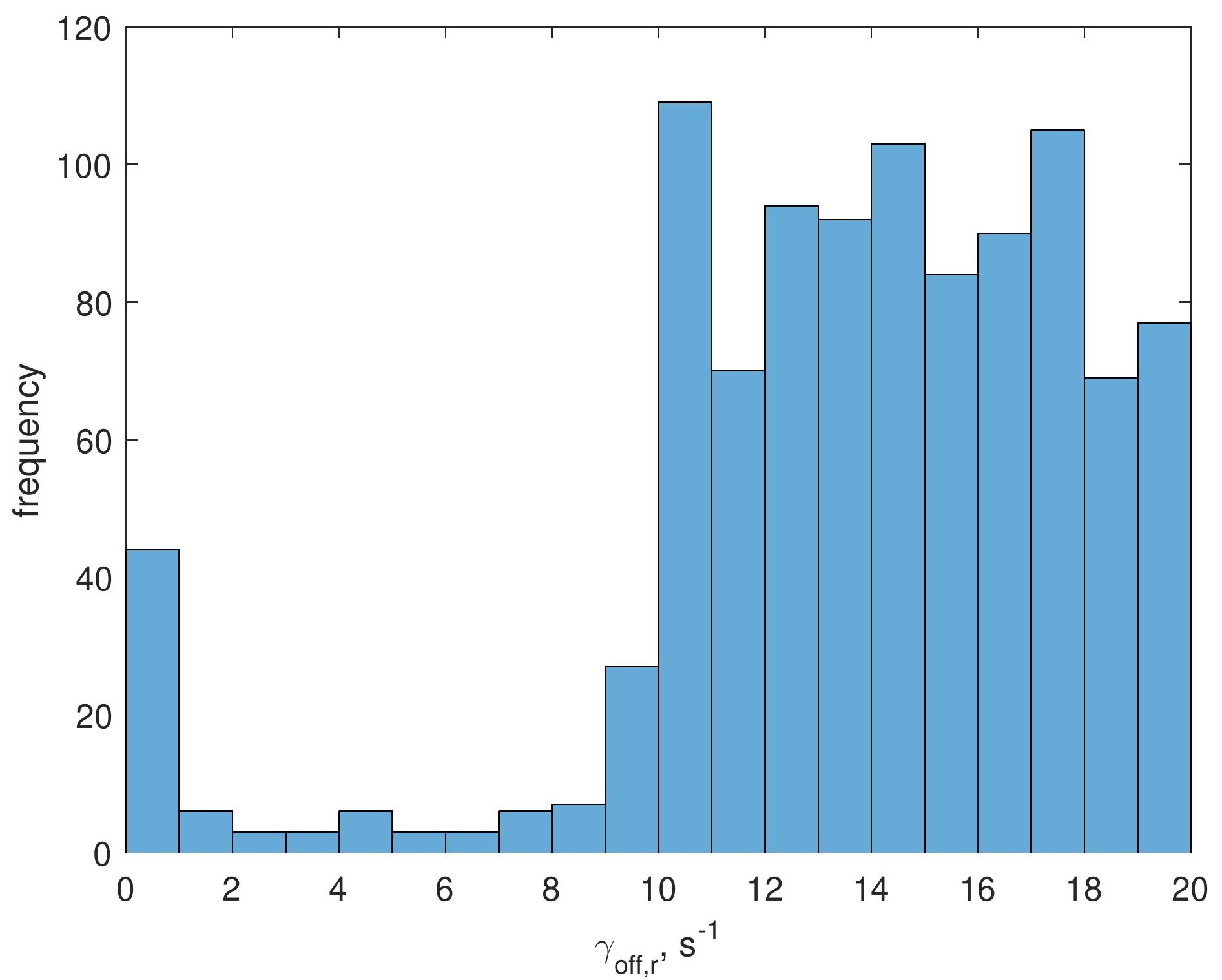




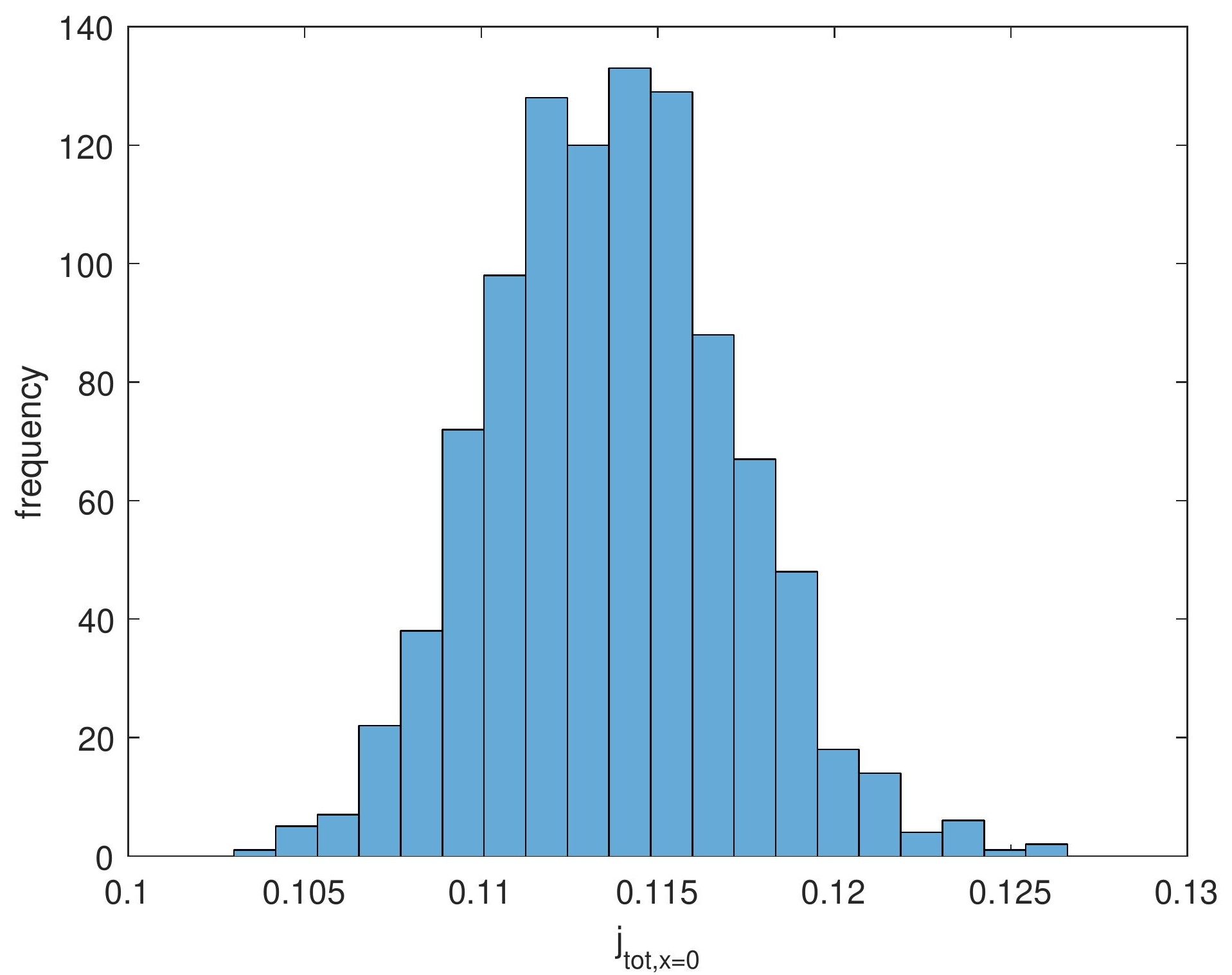




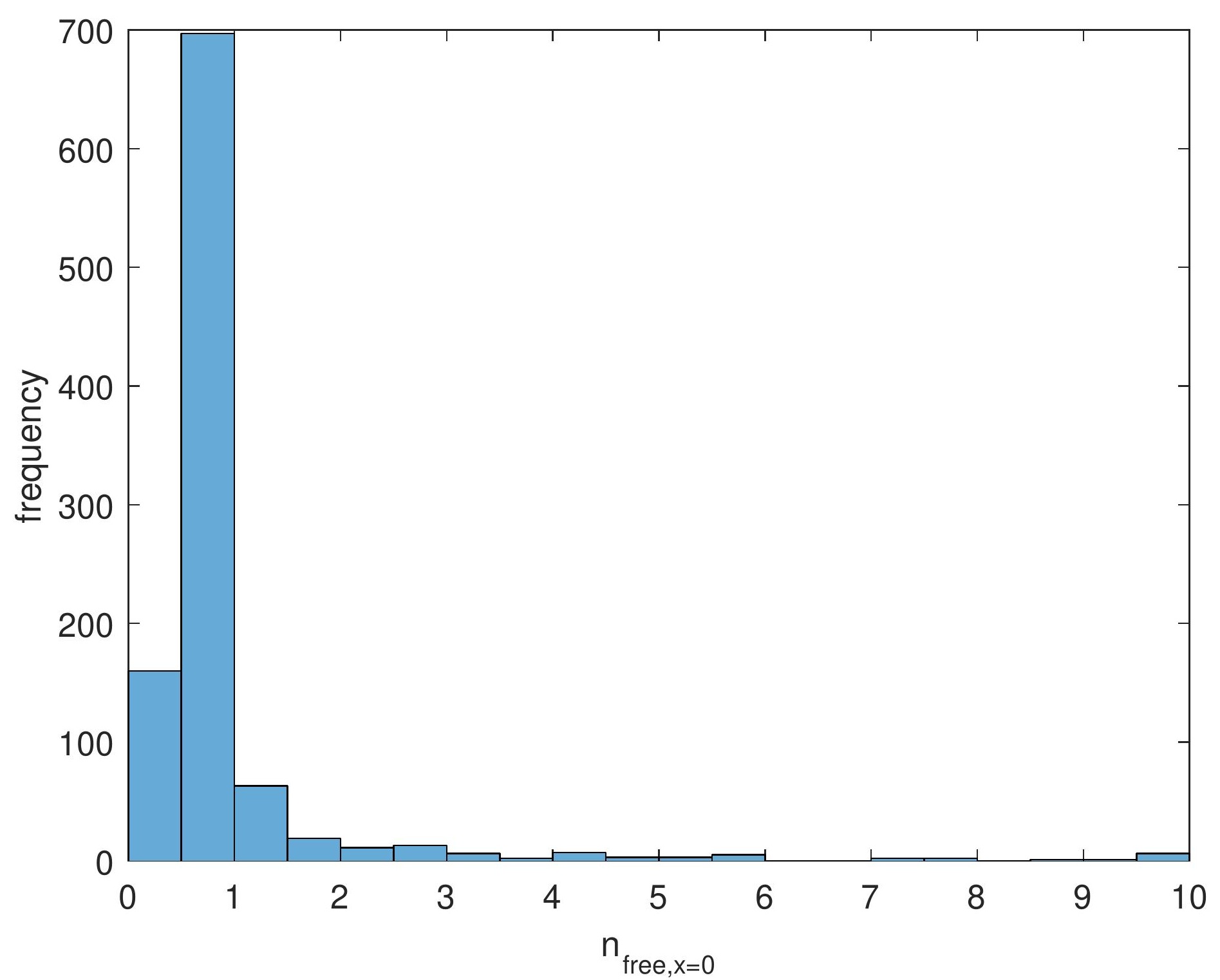




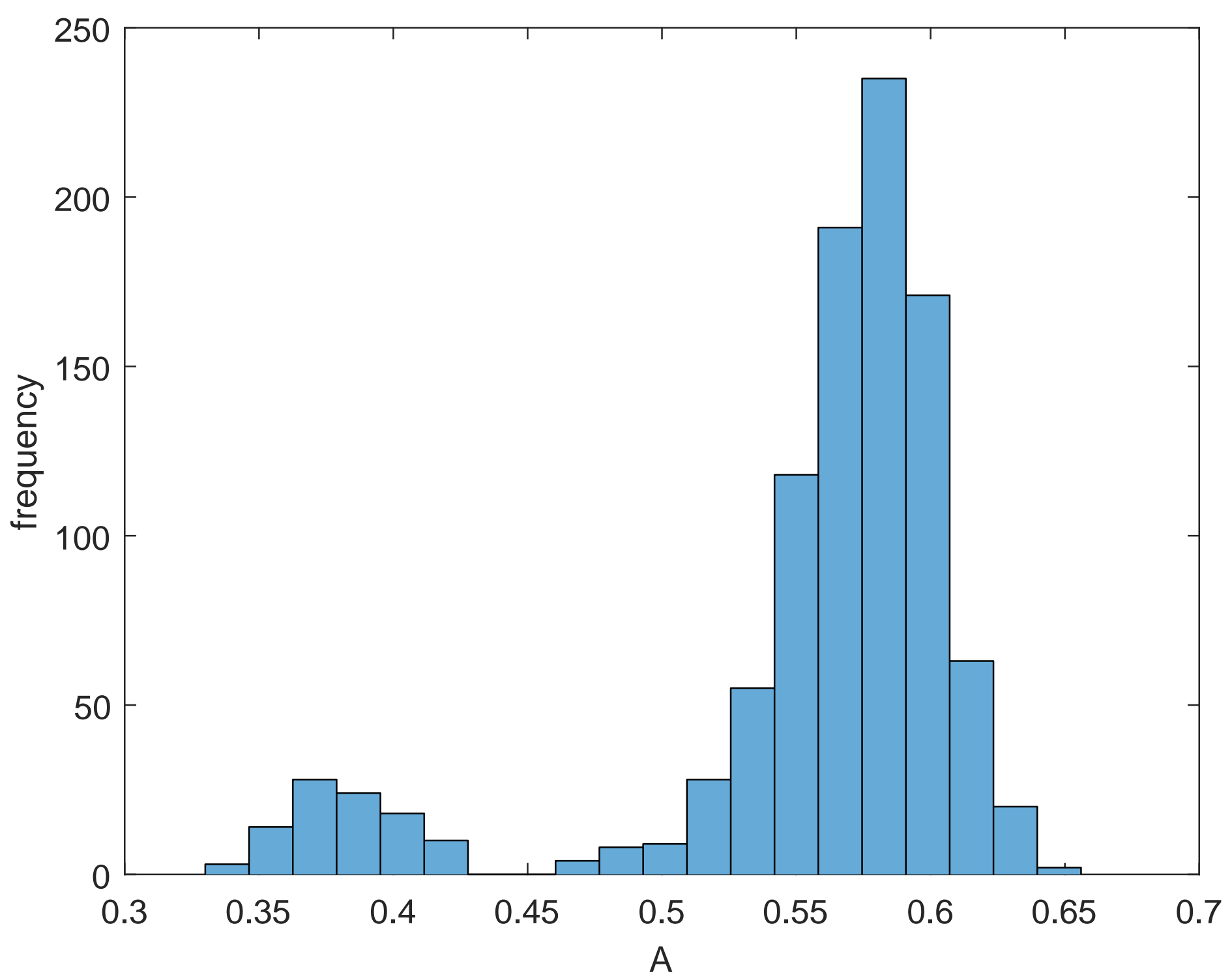

